\title{
A Novel Fault Diagnosis Method for Diesel Engine Based on MVMD and Band Energy
}

\author{
Cheng Gu, Xinyong Qiao (D), Ying Jin, and Yanbin Liu \\ Department of Vehicle Engineering, Academy of Army Armored Force, Beijing, China \\ Correspondence should be addressed to Xinyong Qiao; qxyaafe@sina.com
}

Received 1 February 2020; Accepted 17 June 2020; Published 6 July 2020

Academic Editor: Rafał Burdzik

Copyright $\odot 2020$ Cheng Gu et al. This is an open access article distributed under the Creative Commons Attribution License, which permits unrestricted use, distribution, and reproduction in any medium, provided the original work is properly cited.

\begin{abstract}
Vibration signal, as an important means for diesel engine condition detection and fault diagnosis, has attracted attention for many years. In traditional vibration signal analysis, most processing methods are for single-channel data. However, single-channel vibration signal cannot reflect the operating information of the diesel engine comprehensively because diesel engine vibration is coupled by multiple source signals. This paper proposes the MVMD band energy method for fault diagnosis by four channels of vibration signals. First, the original multivariate signals are decomposed adaptively by MVMD, which obtains a series of components with modal alignment. Then, the band energy values of each measuring point are calculated as the fault characteristics. Finally, SVM is used to realize the diagnosis and identification of diesel engine misfire. The working conditions have a great influence on the vibration signal of the cylinder. In order to obtain the best diagnostic working conditions, six working conditions are set for testing. The result shows that the fault identification rate is highest under the $1500 \mathrm{rpm}$ and $50 \%$ load working condition. The fault recognition rate of this method reaches more than $99 \%$, which is superior to the other four common methods.
\end{abstract}

\section{Introduction}

As the main power source of existing armored vehicles, the efficient and stable operation of diesel engines is an important guarantee for vehicle mobility. With the increasing development of the performance of weapons and equipment, it has put forward higher requirements for the mobility of modern armored vehicles, and diesel engines are required to work continuously and efficiently in the face of complex battlefield environments $[1,2]$. However, the difficult working conditions and harsh working environment lead to the high failure rate of diesel engines. Therefore, it is of great significance to diagnose and eliminate diesel engine failures quickly and accurately to ensure the good operation of armored vehicles.

The cylinder head vibration signal contains wealth information of diesel engine. Compared with other dynamic parameters and thermodynamic parameters, the vibration signal is easy to obtain and can be detected without disassembly. It is widely used in condition monitoring and fault diagnosis. However, the diesel cylinder head vibration signal is usually coupled of vibrations that are generated by multiple excitation sources through a complex transmission path, and the excitation source signal needs to pass through a complicated path to the surface of the cylinder head. It is also mixed with strong background noise. Therefore, the signal has the characteristics of being nonstationary and nonlinear [3-5]. Traditional signal processing methods, such as timedomain signal processing and frequency-domain signal processing, are only suitable for stable and linear signals due to their own characteristics, but they are powerless for nonstationary signals.

In order to solve the shortcomings of traditional signal processing methods, Huang proposed a signal adaptive decomposition method based on the characteristics of the signal itself-empirical mode decomposition (EMD), which can analyze multicomponent coupled signals with nonstationary and nonlinear characteristics [6]. Li applies the EMD method to the collision extraction of abnormal clearance components of diesel engines [7]. Combined with soft 
resampling technology, it can detect clearance-related faults such as valve opening and closing, the wear of piston ring, and bushing. Although EMD can perform adaptive signal decomposition unsupervised, it has many problems such as endpoint effects and modal aliasing, underdecomposition, and overdecomposition. On one hand, aiming at the endpoint effects and modal aliasing shortcomings of EMD, Wu proposed ensemble empirical mode decomposition (EEMD) by adding white noise to the original signal [8], which suppressed the modal aliasing problem to some extent. However, the calculation efficiency is greatly reduced, and false components are separated due to the addition of noise. Wang combined EEMD and fractal dimension to solve the difficulty of diesel engine to identify fault status when two or more fractal dimensions are close to each other [9]. On the other hand, aiming at the underdecomposition and overdecomposition problems of EMD, Smith proposed a new iterative method for demodulating amplitude and frequency modulation signals, namely, local mean decomposition (LMD) [10], but it also has endpoint effects and modal aliasing. Variational Mode Decomposition (VMD) was first proposed by Dragomiretskiy in 2014 [11]. Unlike recursive mode decomposition, it is composed of multiple adaptive Wiener filter banks essentially, which makes its decomposition accuracy and computing efficiency higher, and it can also better solve the problem of modal aliasing in the signal decomposition process. In recent years, more and more scholars have applied VMD and its improved algorithms to fault diagnosis of diesel engines [12-15].

Due to the special working properties, the diesel engine of armored vehicle often has multiple compound faults caused by several parts damaged at the same time. It is impossible to describe the fault characteristics accurately and comprehensively by using only single-channel fault signal [16-18]. With the development of sensor technology, the synchronous acquisition method of multisensor and multichannel has gradually developed, and the multivariate adaptive decomposition method has emerged. The multivariate signal can reflect more information of the mechanical equipment, eliminate the uncertainty of the information to a certain extent, and improve the reliability of the information effectively.

For the processing of multivariate signals, Tanka et al. [19] proposed Complex Empirical Mode Decomposition (CEMD), which essentially uses empirical mode decomposition (EMD) for the real and imaginary parts of complex data, respectively. Based on the correlation between the real part and the imaginary part of the complex data, Rilling et al. [20] proposed Bivariate Empirical Mode Decomposition (BEMD) which gives practical physical significance of each layer component. In 2010, Rehman et al. [21] proposed Trivariate Empirical Mode Decomposition (TEMD) to extend the application of EMD to three-dimensional signals. Subsequently, Rehman et al. projected the multivariate signal to the direction vector of hypersphere in multidimensional space and proposed Multivariate Empirical Mode Decomposition (MEMD) to realize the joint analysis and synchronous processing of multivariate data [22]. Lv applied MEMD to the field of bearing fault diagnosis and proposed a new method for fault feature extraction of rolling bearings based on multivariate EMD [23]. The MEMD solves the problems that multichannel intrinsic mode functions (IMFs) do not match in the number and arrange disorderly on the scale. However, the same shortcoming occurs as in EMD that there are also problems such as endpoint effects and mode aliasing. Mandic et al. [24] proposed Noise-Assisted Multivariate Empirical Mode Decomposition (NAMEMD) by adding Gaussian white noise as an assisted channel. To some extent, the problem of modal aliasing is alleviated. $\mathrm{Wu}$ et al. [25] applied NAMEMD and multiscale morphology to the rolling bearing fault diagnosis, which further reduces the modal aliasing effect and improves the operation speed obviously. This research can extract the fault characteristic frequency of the fault signal clearly and has higher detection accuracy; the fault occurs in the outer ring, inner ring, and roller of the rolling bearing. Although many studies have proposed improved algorithms for multiple EMD, they are essentially based on the EMD algorithm framework, and their defects have not been completely eliminated.

Aiming at the shortcomings of multivariate signals in the decomposition process, Rehman et al. [26] proposed Multivariate Variational Mode Decomposition (MVMD) in 2019, which inherited the advantages of VMD. By constructing a variational optimization problem and extracting the multivariate modulation oscillations inherent in the multivariate signal, this method effectively suppresses the endpoint effect, avoids modal aliasing, and has a solid theoretical basis.

In this paper, 15 types of misfires are diagnosed and identified by MVMD and band energy method. The organization of this paper is arranged as follows: Section 1 introduces the research background and significance; Section 2 studies the MVMD algorithm theory; Section 3 analyzes the simulation signals and compares the advantages and disadvantages of the three methods (MVMD, MEMD, and NAMEMD); Section 4 uses test signals to identify the type of misfire; Section 5 gives conclusions and necessary discusses.

\section{Theory of Multivariate Variational Mode Decomposition}

2.1. Variational Mode Decomposition. In 2014, VMD was proposed by Dragomiretskiy, which solves problems such as modal aliasing during signal decomposition. The goal of VMD is to decompose an input data into a finite and predefined $K$ number of intrinsic principal modes, as equation (1). The decomposition process of VMD algorithm is the solution process of variational problems. The algorithm can be divided into the construction and solution of variational problems:

$$
x(t)=\sum_{k=1}^{K} u_{k}(t) .
$$

2.1.1. Construction of Variational Problems. Assuming that each mode is a finite bandwidth with a center frequency, the variational problem can be described as seeking $K$ mode 
functions, to minimize the sum of the estimated bandwidths of all modes, and the constraint is that the sum of all modes is the original input signal. Firstly, compute the analytic signal of each modal function by Hilbert transform to get its unilateral spectrum; then, modulate the unilateral spectrum of each mode to corresponding base band by adding an estimated center frequency to the analytic signal; finally, the square $L^{2}$ norm of the demodulated signal gradient is calculated to estimate the bandwidth of each modal signal. The variational problem is expressed as

$$
\begin{array}{ll}
\min & \left\{\sum_{k}\left\|\partial_{t}\left[u_{+}^{k}(t) e^{-j \omega_{k} t}\right]\right\|_{2}^{2}\right\} \\
\text { s.t. } & \sum_{k}\left\|u_{k}(t)=x(t)\right\|,
\end{array}
$$

where $u_{+}^{k}(t)$ denotes analytic signal corresponding to $u(t)$ and $\omega_{k}$ denotes the center frequency corresponding to each mode $u(t)$.

2.1.2. Solution of Variational Problems. Firstly, the quadratic penalty factor $\alpha$ and Lagrange multiplication operator $\lambda$ are introduced to change the constrained variational problem into an unconstrained variational problem, where $\alpha$ can guarantee the accuracy of the signal reconstruction in the presence of Gaussian noise and $\lambda$ ensures that the constraints are fulfilled strictly. The resulting augmented Lagrangian function is given as

$$
\begin{aligned}
L\left(\left\{u_{k}\right\},\left\{\omega_{k}\right\}, \lambda\right)= & \alpha \sum_{k}\left\|\partial_{t}\left[u_{+}^{k}(t) e^{-j \omega_{k} t}\right]\right\|_{2}^{2}+\left\|x(t)-\sum_{k} u_{k}(t)\right\|_{2}^{2} \\
& +\left\langle x(t), f(t)-\sum_{k} u_{k}(t)\right\rangle .
\end{aligned}
$$

Then, the alternate direction method of multipliers (ADMM) approach is used to solve the upper variational problem. Using ADMM, the complete optimization problem is divided into a sequence of iterative suboptimization problems.

For example, to update the mode $u_{k}(t)$, the following suboptimization problem is considered at the $n$th iteration:

$$
u_{k}^{n+1} \longleftarrow \underset{u_{k}}{\arg \min } L\left(\left\{u_{i<k}^{n+1}\right\},\left\{u_{i \geq k}^{n}\right\},\left\{\omega_{i}^{n}\right\}, \lambda^{n}\right) .
$$

The above minimization problem can be solved in the spectral domain. The update in the frequency domain is shown as

$$
\widehat{u}_{k}^{n+1}(\omega)=\frac{\widehat{x}(\omega)-\sum_{i \neq k} \widehat{u}_{i}(\omega)+(\widehat{\lambda}(\omega) t / 2)}{1+2 \alpha\left(\omega-\omega_{k}\right)^{2}} .
$$

To update the center frequency $\omega_{k}$, the following suboptimization problem is solved iteratively:

$$
\omega_{k}^{n+1} \longleftarrow \underset{\omega_{k}}{\arg \min } L\left(\left\{u_{i}^{n+1}\right\},\left\{\omega_{1<k}^{n+1}\right\},\left\{\omega_{i \geq k}^{n}\right\}, \lambda^{n}\right) .
$$

The center frequency $\omega_{k}^{n+1}$ can be updated in the dual frequency domain, which estimates the new frequency as the center of gravity of the associated modes' power spectrum:

$$
\omega_{k}^{n+1}=\frac{\int_{0}^{\infty} \omega\left|\widehat{u}_{k}(\omega)\right|^{2} \mathrm{~d} \omega}{\int_{0}^{\infty}\left|\widehat{u}_{k}(\omega)\right|^{2} \mathrm{~d} \omega} .
$$

2.2. Multivariate Variational Mode Decomposition. MVMD has recently been proposed to solve the problem of multichannel data collaborative decomposition to improve VMD which can only process single-channel data. As a generalized extension of the original VMD algorithm, the main goal of MVMD is to extract multivariate modulated oscillations $\mathbf{u}_{k}(t)$ from input data $\mathbf{x}(t)$ containing $C$ number of data channels. The number of multivariate modulated oscillations is preset to $K$ :

$$
x(t)=\sum_{k=1}^{k} u_{k}(t)
$$

where $u_{k}(t)=\left[u_{1}(t), u_{2}(t), \ldots, u_{C}(t)\right]$ and $x(t)=\left[x_{1}(t)\right.$, $\left.x_{2}(t), \ldots, x_{C}(t)\right]$.

The following two conditions need to be met when the MVMD decomposition is completed.

(i) The sum of bandwidths of the extracted modes is minimum

(ii) The sum of the extracted modes recovers the original signal $u_{k}(t)$

The construction of variational problem is similar to VMD. The variational problem is expressed as equation (9). Two important points should be pointed out in a multivariate extension. One is that the vector analytic representation of $\mathbf{u}_{k}(t)$ denoted by $\mathbf{u}_{+}^{k}(t)$ and the bandwidth of $\mathbf{u}_{k}(t)$ can be estimated by taking the $L_{2}$ norm of the gradient function of the harmonically shifted $\mathbf{u}_{+}^{k}(t)$. The other is that the bandwidth of modulated multivariate oscillation is estimated by shifting the unilateral frequency spectrum of all channels of $\mathbf{u}_{+}^{k}(t)$ by $\omega_{k}$ and taking the Frobenius norm ${ }^{1}$ of the resulting matrix, in order to find multivariate oscillations in $\mathbf{u}_{k}(t)$ :

$$
\begin{array}{ll}
\min & \left\{\sum_{k} \sum_{c}\left\|\partial_{t}\left[u_{+}^{k, c}(t) e^{-j \omega_{k} t}\right]\right\|_{2}^{2}\right\} \\
\text { s.t. } & \sum_{k} u_{k, c}(t)=x_{c}(t), \quad c=1,2, \ldots, C,
\end{array}
$$

where $u_{k}(t)$ denotes the analytic modulated signal corresponding to channel number $c$ and mode number $k$.

In the process of solving multiple variational problem, since multiple linear equation constraints correspond to the 
total number of channels, the corresponding augmented Lagrangian function becomes

$$
\begin{aligned}
L\left(\left\{u_{k, c}\right\},\left\{\omega_{k}\right\}, \lambda_{c}\right)= & \alpha \sum_{k} \sum_{c}\left\|\partial_{t}\left[u_{+}^{k, c}(t) e^{-j \omega_{k} t}\right]\right\|_{2}^{2} \\
& +\sum_{c}\left\|x_{c}(t)-\sum_{k} u_{k, c}(t)\right\|_{2}^{2} \\
& +\sum_{c}\left\langle\lambda_{c}(t), x_{c}(t)-\sum_{k} u_{k, c}(t)\right\rangle .
\end{aligned}
$$

The above unconstrained optimization problem is solved using ADMM approach. This includes mode update and center frequency update in variational problem solving process. Corresponding to equations (4-7) in VMD, the solution of variational problem in MVMD becomes equations (11-14). The steps are listed in Algorithm 1. We refer readers to reference [26] for details:

$$
\begin{aligned}
u_{k, c}^{n+1} & =\underset{u_{k, c}}{\arg \min }\left\{\alpha\left\|\partial_{t}\left[u_{+}^{k, c}(t) e^{-j \omega_{k} t}\right]\right\|_{2}^{2}+\left\|x_{c}(t)-\sum_{i} u_{i, c}(t)+\frac{\lambda_{c}(t)}{2}\right\|_{2}^{2}\right\}, \\
\widehat{u}_{k, c}^{n+1}(\omega) & =\frac{\widehat{x}_{c}(\omega)-\sum_{i \neq k} \widehat{u}_{i, c}(\omega)+\left(\hat{\lambda}_{c}(\omega) / 2\right)}{1+2 \alpha\left(\omega-\omega_{k}\right)^{2}}, \\
\omega_{k}^{n+1} & =\underset{\omega_{k}}{\arg \min }\left\{\sum_{c}\left\|\partial_{t}\left[u_{+}^{k, c}(t) e^{-j \omega_{k} t}\right]\right\|_{2}^{2}\right\}, \\
\omega_{k}^{n+1} & =\frac{\sum_{c} \int_{0}^{\infty} \omega\left|\widehat{u}_{k, c}(\omega)\right|^{2} \mathrm{~d} \omega}{\sum_{c} \int_{0}^{\infty}\left|\widehat{u}_{k, c}(\omega)\right|^{2} \mathrm{~d} \omega} .
\end{aligned}
$$

\section{Analysis of Simulation Signal}

3.1. Construction of Simulation Signal. The diesel engine has the complex internal structure with many parts, and the vibration of the excitation source needs to pass a certain transmission path before the sensor receives the signal. Generally, the vibration signals collected by sensors are nonlinear and nonstationary modulation signals. According to the actual working conditions, the amplitude modulation signal $x_{1}$, the frequency modulation signal $x_{2}$, and the harmonic signal $x_{3}$ are, respectively, set to simulate the original signal of the diesel engine in the simulation experiment, as shown in equation (15). The frequencies of the simulation signal are as follows: $f_{1}=15 \mathrm{~Hz}, f_{2}=100 \mathrm{~Hz}$, $f_{3}=80 \mathrm{~Hz}, f_{4}=10 \mathrm{~Hz}, f_{5}=50 \mathrm{~Hz}$ :

$$
\begin{aligned}
& x_{1}=\left[1+\cos \left(2 \pi f_{1} t\right)\right] \sin \left(2 \pi f_{2} t\right), \\
& x_{2}=\sin \left(2 \pi f_{3} t+\cos \left(2 \pi f_{4} t\right)\right), \\
& x_{3}=\cos \left(2 \pi f_{5} t\right) .
\end{aligned}
$$

In the actual acquisition process, the vibration signals collected from different position sensors have different signal transmission path and noise interference. Based on the above considerations, the simulation signals are composed of three original signals with different amplitude weights, and white noise is added to them. The noise of the three channels is random Gaussian white noise with a mean of 0 and a standard deviation of 0.1 . The multivariate simulation signals are shown in

$$
\begin{aligned}
& s_{1}(t)=0.6 x_{1}(t)+x_{2}(t)+0.9 x_{3}(t)+n_{1}, \\
& s_{2}(t)=x_{1}(t)+x_{2}(t)+0.7 x_{3}(t)+n_{2}, \\
& s_{3}(t)=0.7 x_{1}(t)+1.2 x_{2}(t)+0.8 x_{3}(t)+n_{3} .
\end{aligned}
$$

The time-domain and frequency-domain waveforms of the simulation signal are shown in Figure 1. It can be seen from the figure that the time-domain signals of the three channel signals are more chaotic, and the internal laws cannot be found. There are multiple peaks in the frequencydomain waveform. The characteristic frequency components of the simulated signal are reflected, but the sideband amplitude is high, and the characteristic frequency is not clear.

3.2. Performance Comparison. In order to show the superiority of MVMD in adaptive decomposition, MEMD, NAMEMD, and MVMD were used to decompose the simulation signals and the results were compared. Among them, in NAMEMD, a two-channel Gaussian white noise signal is added to the simulation signal for decomposition, in order to reduce the modal aliasing and highlight the characteristic frequency. The white noise signal is the same length as the original signal. In MVMD, the number of modes $K$ is set to 4 , and the penalty factor is set to 2000 .

The results obtained by decomposing the simulation signals using three multivariate decomposition algorithms are as follows. 12 sets of IMF components are obtained by MEMD, and characteristic frequencies are mainly 


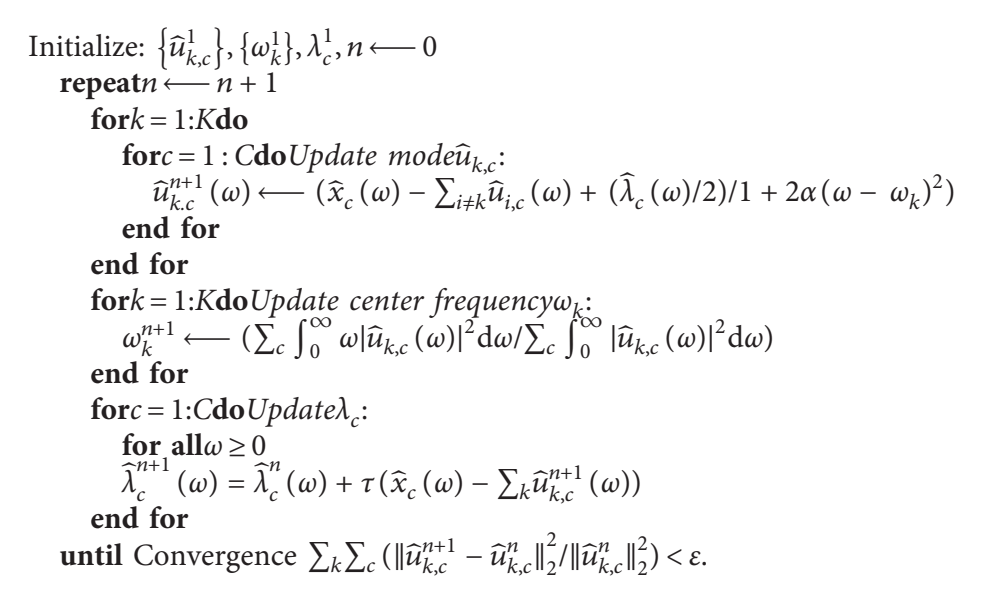

Algorithm 1: Multivariate Variational Mode Decomposition.

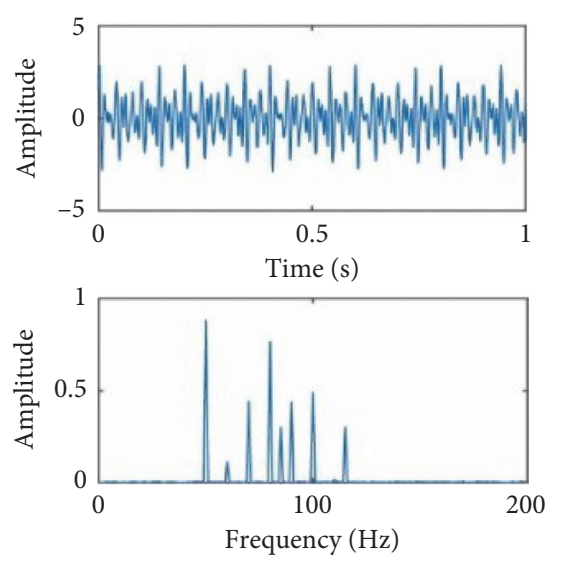

(a)

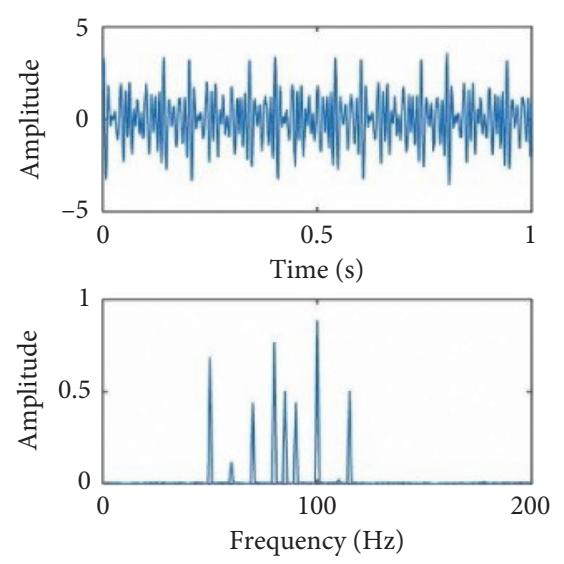

(b)

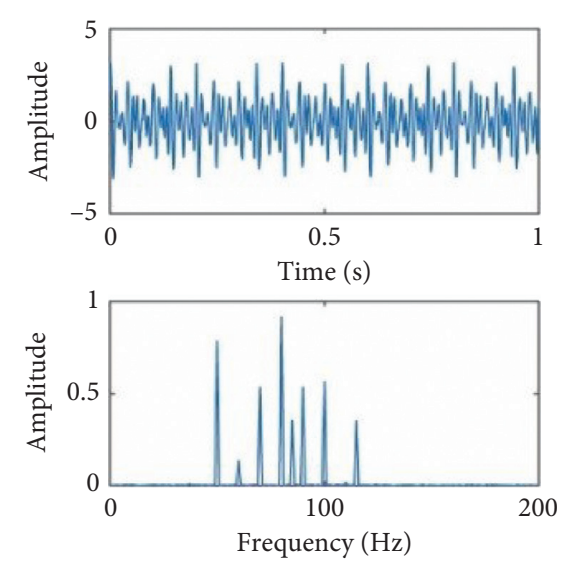

(c)

Figure 1: The time domain and frequency domain waveforms of the simulation signal. (a) Simulation signal $s_{1}$. (b) Simulation signal $s_{2}$. (c) Simulation signal $s_{3}$.

distributed in the 4 th- to 6th-order IMF groups through spectrum analysis. Therefore, the 4th- to 6th-order IMF groups are characteristic IMF groups. The time domain and frequency domain waveforms are shown in Figure 2. Similarly, 12 sets of IMF components are obtained by NAMEMD, and characteristic frequencies are also distributed in the 4th- to 6th-order IMF groups. The time domain and frequency domain waveforms are shown in Figure 3. Due to $K=4$, the decomposition result by MVMD obtains 4 sets of IMF components, and characteristic frequencies are mainly distributed in the 1st- to 3rd-order IMF groups through spectrum analysis. Therefore, the 1st- to 3rd-order IMF groups are characteristic IMF groups. The time domain and frequency domain waveforms are shown in Figure 4. In addition, the calculation time of the three algorithms is shown in Table 1.

As can be seen from the frequency-domain waveforms in Figures 2-4, three algorithms can adaptively decompose the multivariate simulation signals to obtain IMF components that can reflect the characteristic frequency. And the same frequency component of different channels is reflected in the same order IMF, namely, mode alignment. However, the modal aliasing phenomenon is more serious in the characteristic IMF group obtained by MEMD. Meanwhile, the following problems exist, such as the large sideband amplitude and the obvious noise component. The characteristic frequency $f_{3}$ appears in the 4 th- and 5 th-order characteristic IMF groups at the same time, and the characteristic frequency $f_{5}$ appears in the 5 th- and 6th-order characteristic IMF groups at the same time. The sidebands of the amplitude modulation signal $x_{1}$ and the frequency modulation signal $x_{2}$ are obvious, and the harmonic signals $x_{3}$ are also affected that a frequency component of $35 \mathrm{~Hz}\left(f_{5}-f_{1}\right)$ appears in IMF6. In the characteristic IMF groups obtained by the NAMEMD decomposition, modal aliasing is reduced and noise component is also improved as the noise of two channels is added to participate in the decomposition at the same time. The characteristic frequency is relatively more 

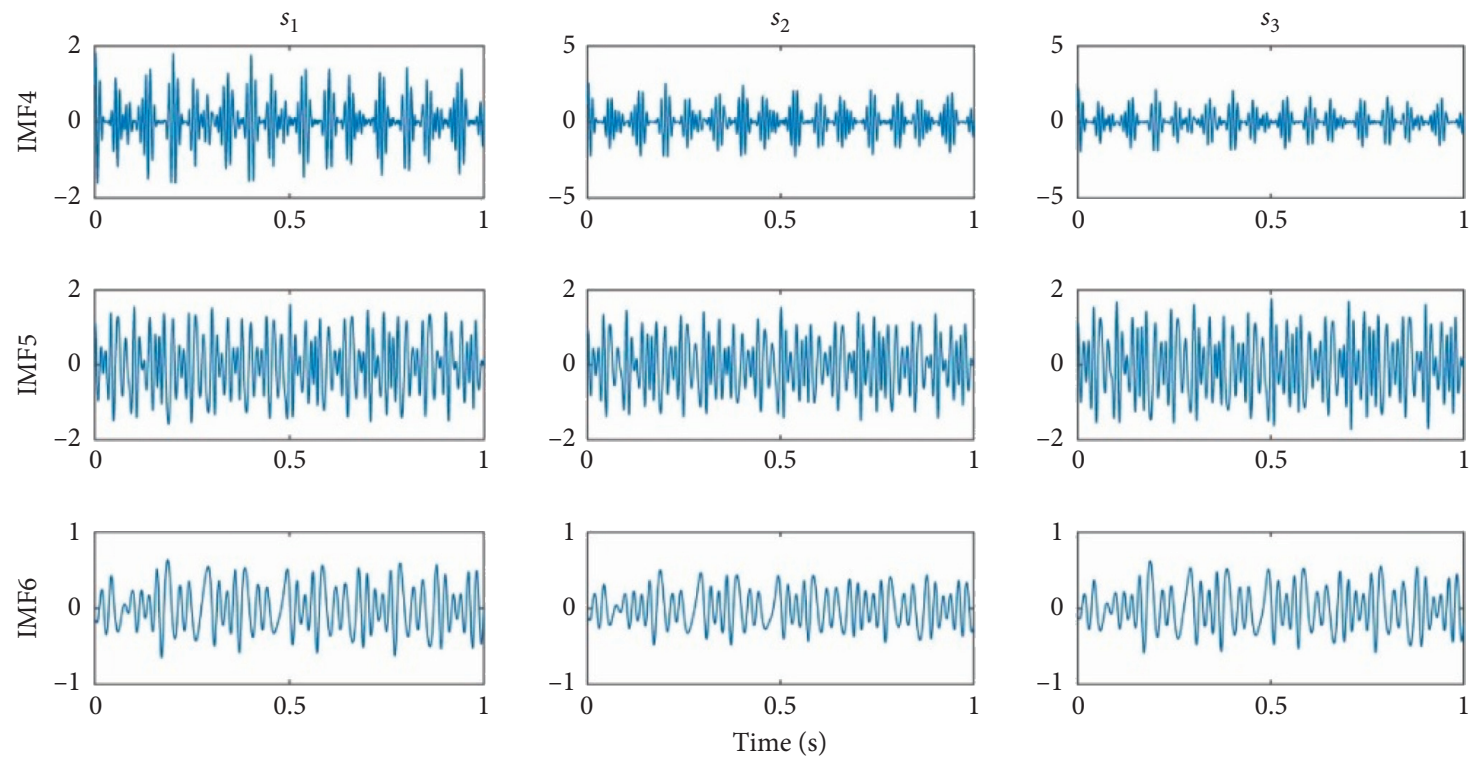

(a)
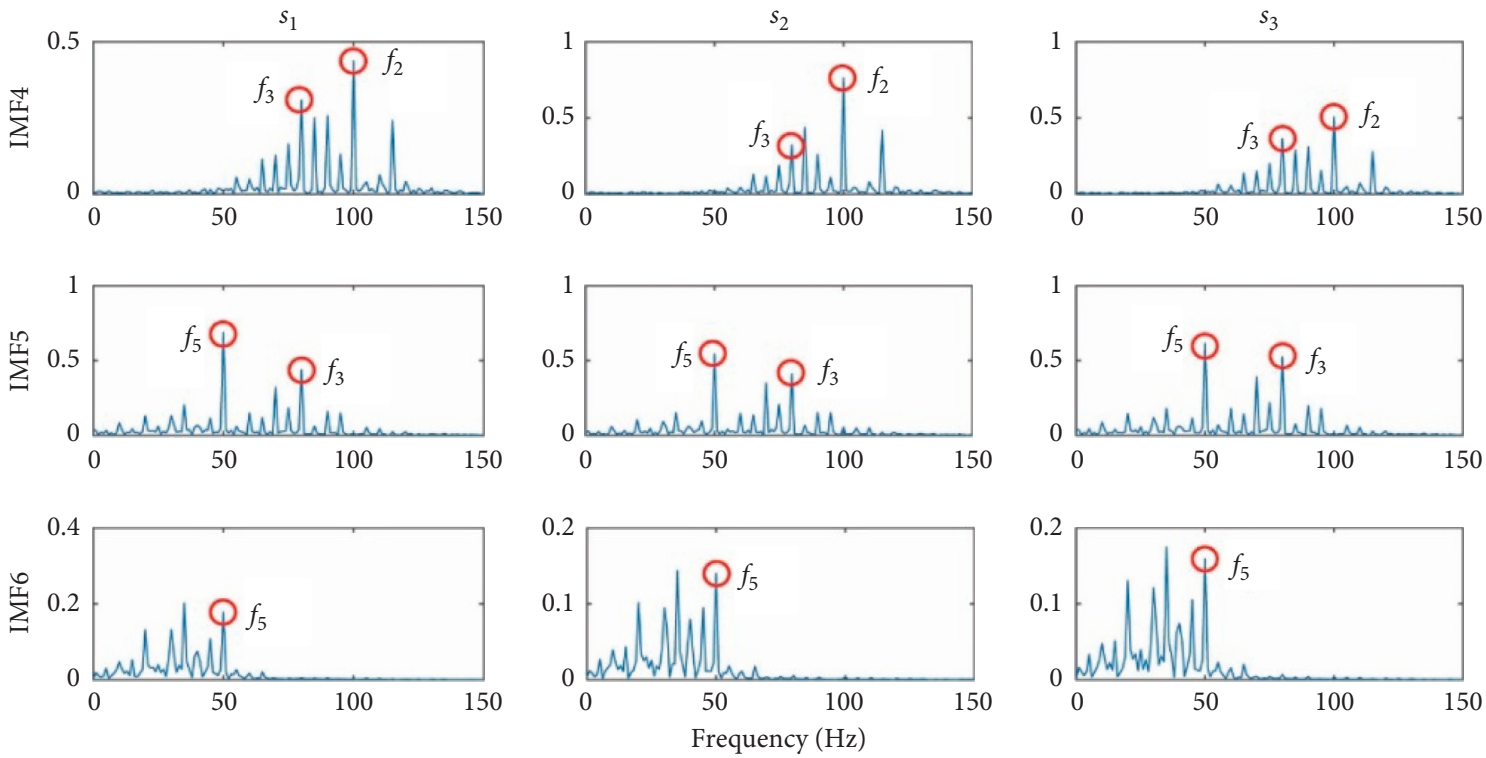

(b)

Figure 2: The decomposition results by MEMD (IMF4-IMF6). (a) Time domain. (b) Frequency domain.

prominent than that of MEMD, but the deficiency of MEMD is not completely overcome. In the characteristic IMF groups obtained by the MVMD decomposition, the first three orders of the IMF groups, respectively, reflect the frequency components of $x_{3}, x_{2}$, and $x_{1}$, and the amplitude of the characteristic frequency is more clear. The phenomenon of modal aliasing disappears, and there are only some sideband components with small amplitude. What is more, noise signals are also suppressed. It can be seen from the above analysis that the decomposition effect of MVMD is superior to that of MEMD and NAMEMD. Comparing the calculation time of the three algorithms, it is concluded that the calculation time of MVMD is shorter and the calculation efficiency is higher.

\section{Analysis of Test Signal}

4.1. Scheme of Fault Diagnosis. Misfire is one of common failure types of diesel engine, which affects the dynamic performance of the diesel engine severely. The traditional identification method of misfire fault is by analyzing single-channel vibration data from the acceleration sensor. The acceleration sensor is fixed at a certain position on the cylinder head of the diesel engine. However, as a complex power machine, diesel engine has many excitation sources. The vibration signals generated by the excitation sources are transmitted to the cylinder head surface through complicated path, which makes the collected vibration signals appear nonstationary and 

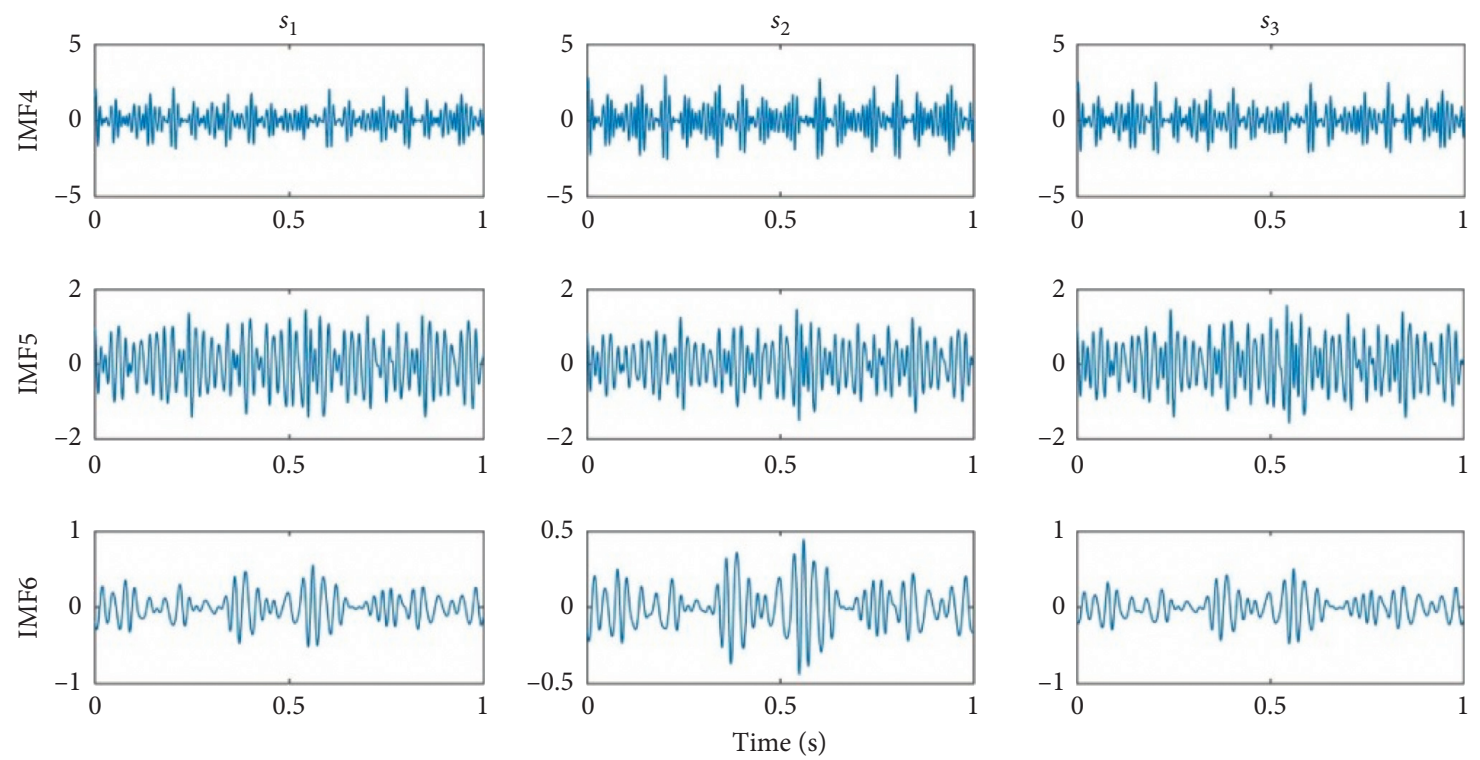

(a)
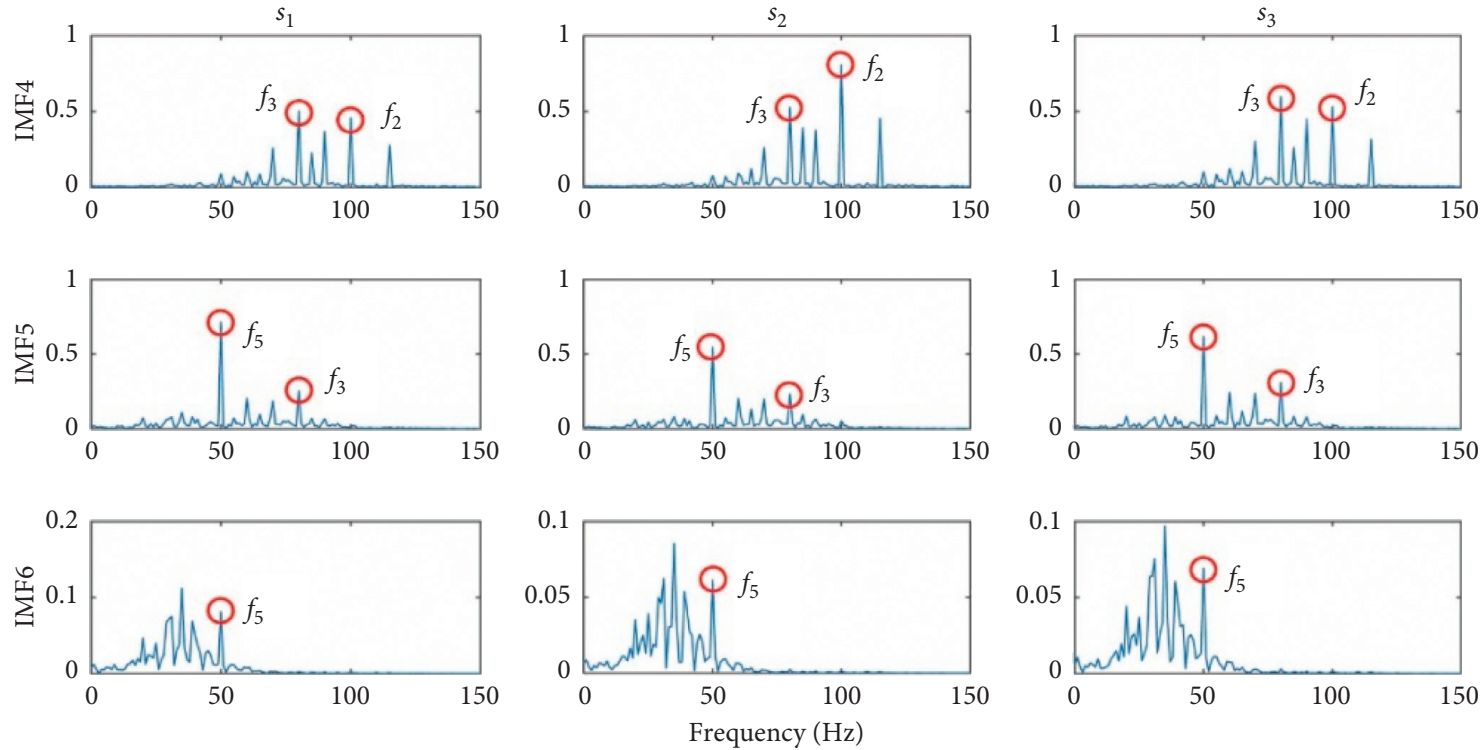

(b)

Figure 3: The decomposition results by NAMEMD (IMF4-IMF6). (a) Time domain. (b) Frequency domain.

nonlinear. Moreover, the vibration data of single channel often cannot describe the fault characteristics accurately and comprehensively, resulting in a low recognition rate of misfire faults.

In view of the above shortcomings of single-channel signal, this paper proposes a diesel engine fault diagnosis method based on MVMD and multipoint band energy, which can find the location of the misfire cylinder quickly and accurately. The flowchart is shown in Figure 5. Firstly, the number of modes $K$ is determined by the center frequency method, and the original vibration signals are decomposed into a series of modal aligned components by MVMD adaptively. Then, the band energy value of each decomposed component is calculated as the fault characteristic. Finally, support vector machine (SVM) is used to realize the diagnosis and identification of the diesel engine misfire.

4.2. Bench Test. As the research object for the bench test, a 12-cylinder exhaust turbocharged diesel engine with fourstroke was used to generate vibration signals. The main parameters of the diesel engine are as follows: cylinder bore $(150 \mathrm{~mm})$, connecting rod length $(180 \mathrm{~mm})$, compression ratio (13-14), and rated power/speed (588 kW/2200 rpm). The firing order of the diesel engine cylinder is shown in Figure 6. The test system is shown in Figure 7, which mainly includes a diesel engine bench, a collection system, a 

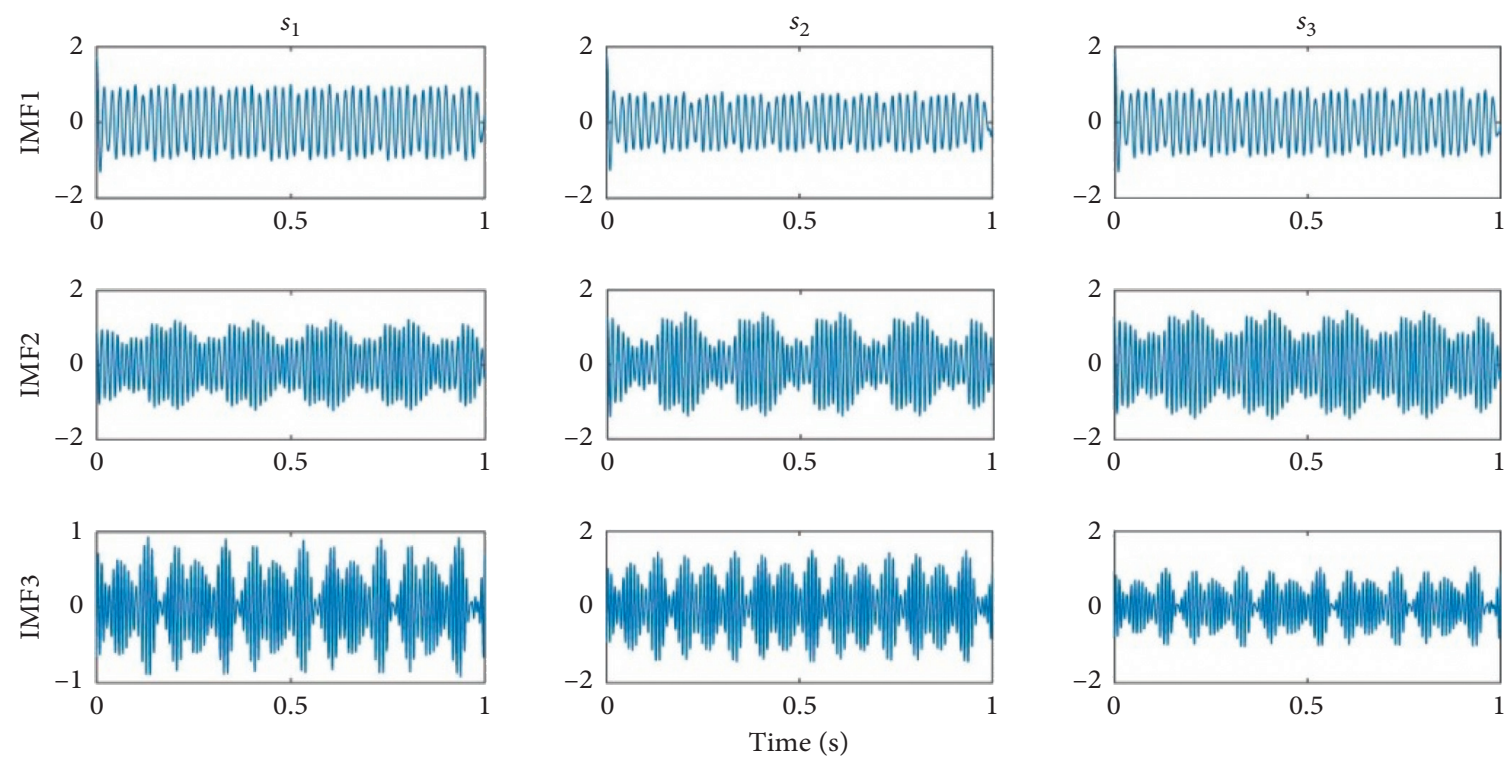

(a)
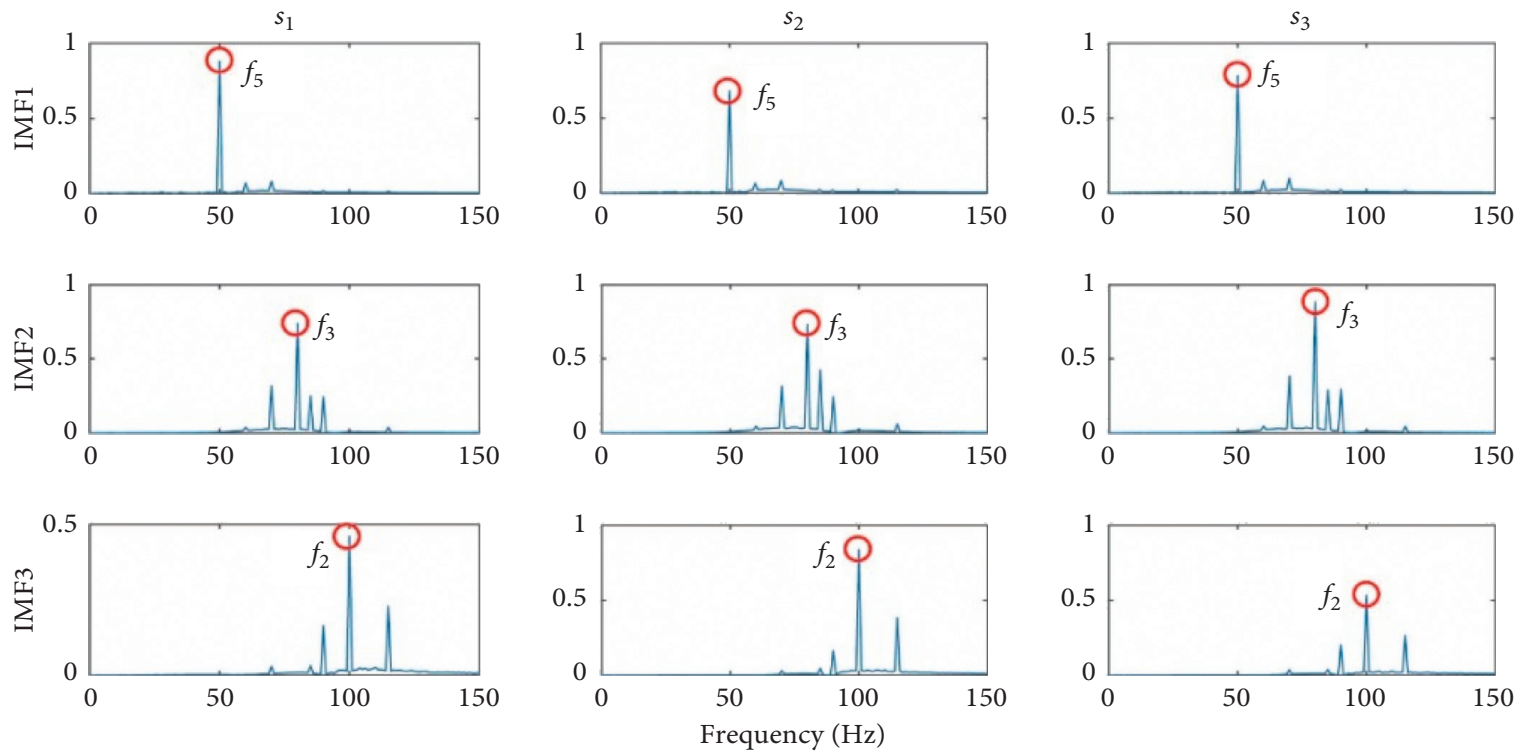

(b)

Figure 4: The decomposition results by MVMD (IMF1-IMF3). (a) Time domain. (b) Frequency domain.

TABle 1: The calculation time of the three algorithms.

\begin{tabular}{lccc}
\hline Algorithm & MEMD & NAMEMD & MVMD \\
\hline Time $(\mathrm{s})$ & 70.9 & 39.8 & 7.2 \\
\hline
\end{tabular}

dynamometer, and a computer. When installing the vibration acceleration sensor, four measuring points are arranged at the two ends of the diesel engines for signal acquisition after comprehensive consideration, namely, the left 1-cylinder head (measuring point 1), the left 6-cylinder head (measuring point 2 ), the right 1 -cylinder head (measuring point 3 ), and the right 6-cylinder head (measuring point 4), as shown in Figure 8. The sensitivity of the acceleration sensor is $10 \mathrm{mV} /\left(\mathrm{m} / \mathrm{s}^{2}\right)$.
During bench test, the cylinder misfire fault was simulated by disconnection of the high-pressure oil pipe and the injector. Different misfire faults, such as single-cylinder misfire fault and multicylinder misfire fault, were set, respectively. The specific settings are shown in Table 2. The operating conditions (speed and load) of the diesel engine have a greater impact on the cylinder vibration signal. In order to obtain the best working conditions for diagnosis, six working conditions are set for test, including no load (800 rpm, $1500 \mathrm{rpm}, 2200 \mathrm{rpm})$ and 50\% load (800 rpm, $1500 \mathrm{rpm}, 2200 \mathrm{rpm})$. The sampling frequency was set as $20 \mathrm{kHz}$, and the sampling time was set as $0.5 \mathrm{~s}$. 60 sets of vibration signals were collected under each working condition. 


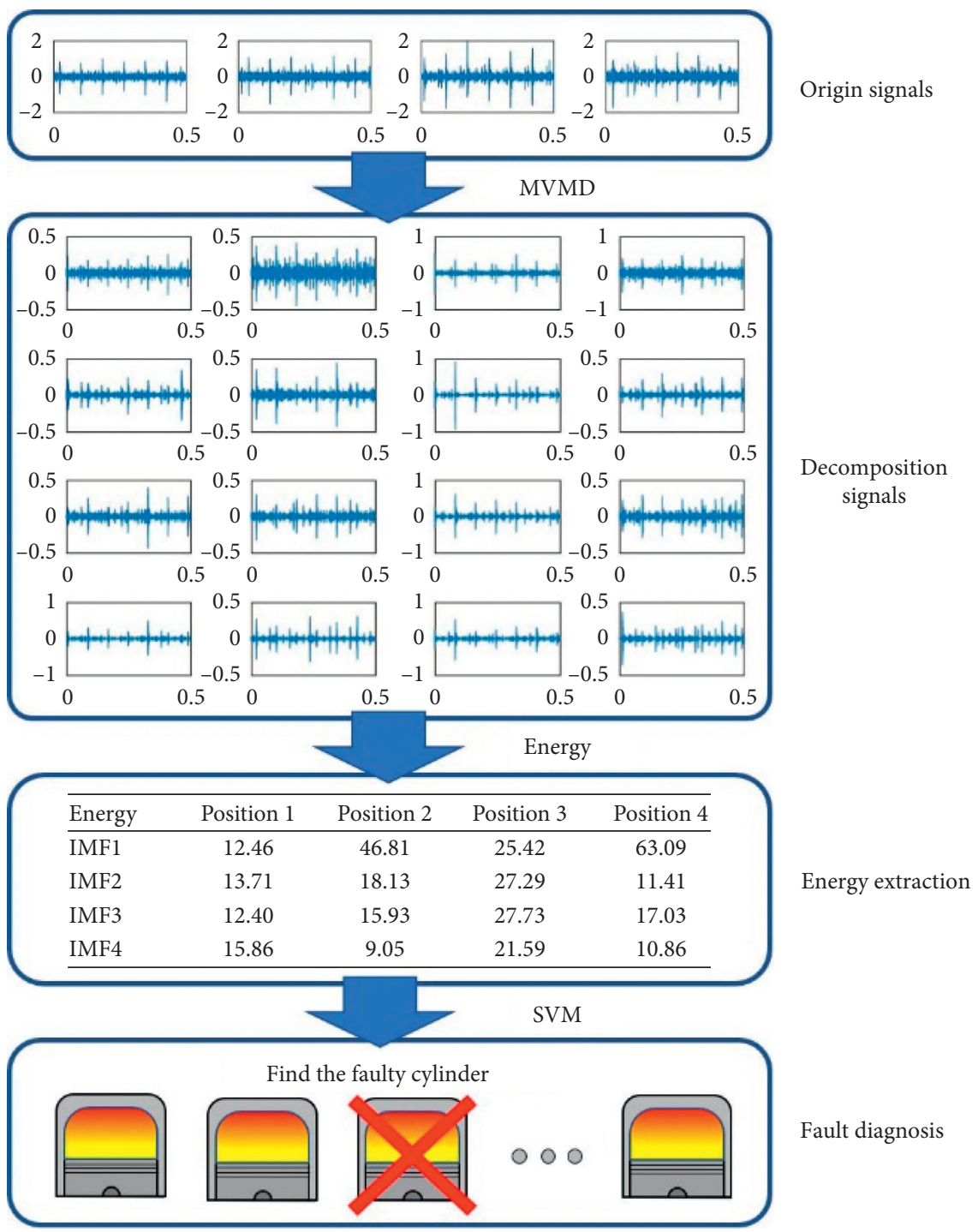

FIgURE 5: Fault diagnosis flowchart of diesel engine misfire.

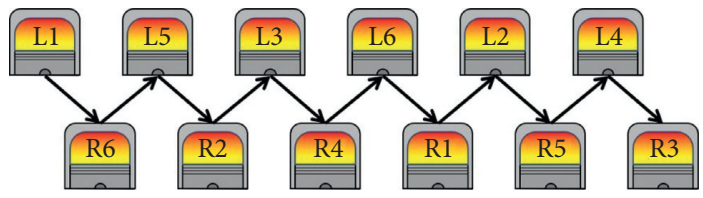

Figure 6: The firing order of 12-cylinder diesel engine.

\subsection{Signal Processing and Fault Diagnosis}

4.3.1. Extraction of Band Energy. Combustion excitation, as the main excitation source of diesel engines, directly affects the response characteristics of cylinder head vibration signals. Misfire failure, no combustion in a cylinder, not only changes the amplitude of the vibration signal in the time domain, but also changes its frequency domain distribution. Considering the significant impact of speed and load on combustion, combustion burst is moderate at low-speed and low-load condition, and the vibration signal is relatively small. In contrast, the severe combustion burst at high-speed

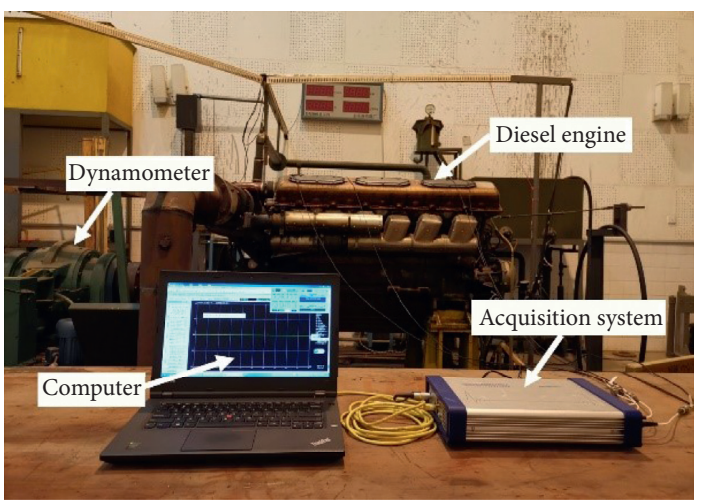

Figure 7: Bench of diesel engine.

and high-load leads to the vibration signal increasing. Therefore, in the diagnosis of diesel engine misfire faults, the characteristic parameters of each fault state should be compared under the same operating conditions. In order to 


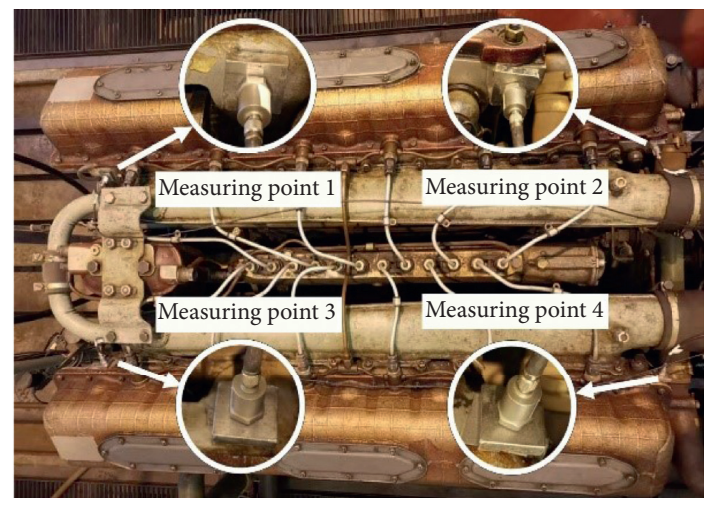

Figure 8: The measuring points.

TABle 2: Description of the cylinder state.

\begin{tabular}{lcc}
\hline No. $x$ & Misfire cylinder & Fault type \\
\hline 1 & None & Normal \\
2 & L1 & Single cylinder \\
3 & L2 & Single cylinder \\
4 & L3 & Single cylinder \\
5 & L4 & Single cylinder \\
6 & L5 & Single cylinder \\
7 & L6 & Single cylinder \\
8 & R1 & Single cylinder \\
9 & R2 & Single cylinder \\
10 & R3 & Single cylinder \\
11 & R4 & Single cylinder \\
12 & R5 & Single cylinder \\
13 & R6 & Single cylinder \\
14 & L1 \& R6 & Neighbor cylinder \\
15 & L1 \& L4 & Far cylinder \\
\hline
\end{tabular}

facilitate the research, the following research will be carried out on the $1500 \mathrm{rpm}$ and $50 \%$ load condition if there is no special explanation. Owing to many fault states involved, this paper only uses normal working state and left 1-cylinder misfire as examples for analysis during the fault feature parameter extraction stage.

The time-domain and frequency-domain waveforms of original signals under normal state are shown in Figure 9. The time domain and frequency domain waveforms of original signals under left 1 -cylinder misfire state are shown in Figure 10. Comparing the two figures, it can be seen that each measuring point has a relatively regular impact peak under normal state. However, in the fault state, the main excitation source is lacking during the explosion phase of the left 1-cylinder due to the misfire of the left 1-cylinder, and there is no obvious impact peak at measuring point 1 . Similarly, the frequency amplitude of measuring point 1 also decreases to some extent. So the difference between the normal state and the left 1-cylinder misfire fault can be distinguished from the time domain analysis and frequency domain analysis. The reason is that the misfire cylinder (L1) is located at the installation position of measuring point 1 . The combustion excitation can be directly transmitted to the acceleration sensor through the cylinder wall, and the transmission path is simple. While the method of the time domain analysis and the frequency domain analysis of the vibration signal fails to distinguish the difference of normal state and left 3-cylinder misfire, or left 1-cylinder misfire and left 1-cylinder \& left 4-cylinder misfire, further processing of the multichannel signal is required.

When a cylinder misfire fault occurs, the response of the vibration signal will change due to the change in the excitation. Furthermore, impacts of misfire faults are differentiated by various frequency bands at each measuring point. Different frequency bands can be extracted by MVMD from multivariate signals, so MVMD is used to decompose original signals collected from cylinder head. Firstly, the number of modes $K$ needs to be determined. $K$ value has a greater impact on the decomposition effect. If $K$ value is too small, the multivariate signals are underdecomposed; if $K$ value is too large, the multivariate signals are overdecomposed, and false components are decomposed. Both of the above situations would affect subsequent analysis. In order to determine appropriate $K$ value, $K=3 / 4 / 5 / 6$ is selected to perform MVMD on the vibration signals of the normal state and the left-cylinder misfire fault, respectively. The center frequency distribution of 60 samples at different $K$ values is shown in Figures 11 and 12. It can be seen from the figure that as the value of $\mathrm{K}$ increases, the center frequency distribution of the two states is basically the same. When $K<4$, it is an underdecomposition condition. When $K>4$, the center frequency waveform appears to oscillate violently or be close between two components; it is an overdecomposition condition. Therefore, it is appropriate to select the number of modes $K=4$. After analysis, the optimal number of modes for each of the 15 types of misfire states in Table 1 is 4 .

The decomposition results under two states by MVMD at $K=4$ are shown in Figures 13 and 14. It is difficult to judge the fault status from the time domain waveform directly. From the view of energy, the band energy values of different frequency at each measuring points are extracted as the fault characteristic parameter. The energy values under the normal state and the left 1-cylinder misfire fault are shown in Table 3; it can be seen that the misfire of left 1-cylinder has different effects on the energy values of the four measuring points in multiple frequency bands.

4.3.2. Misfire Fault Diagnosis. Support vector machine (SVM) is an intelligent classifier based on the principle of minimizing structural risks. The optimal classification mode can be searched under small sample conditions, which effectively avoids the problems of underlearning, overlearning, and local minima in neural network algorithms. Therefore, it is very suitable for fault diagnosis of rotating machinery under small sample conditions.

According to the above method, the band energy values of 15 states are calculated as the input vector of the SVM, and the labels of the 15 states are set to $1-15$ in the order of Table 1 . There are 60 sets of samples in each state. 48 sets are randomly selected as training samples and the other 12 groups are used as test samples. Therefore, there are a total of 720 training samples and a total of 120 test samples. The 

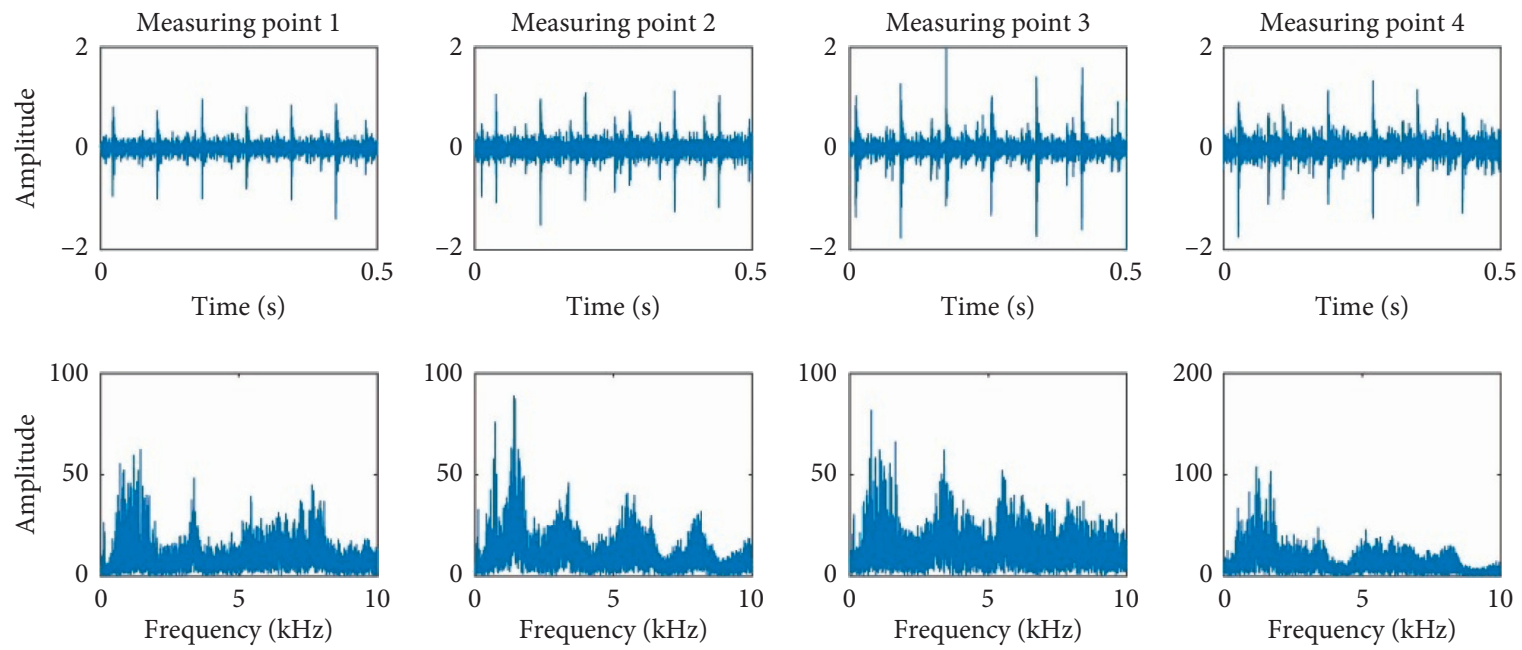

FIGURE 9: The waveforms of original signals under normal state (time domain and frequency domain).
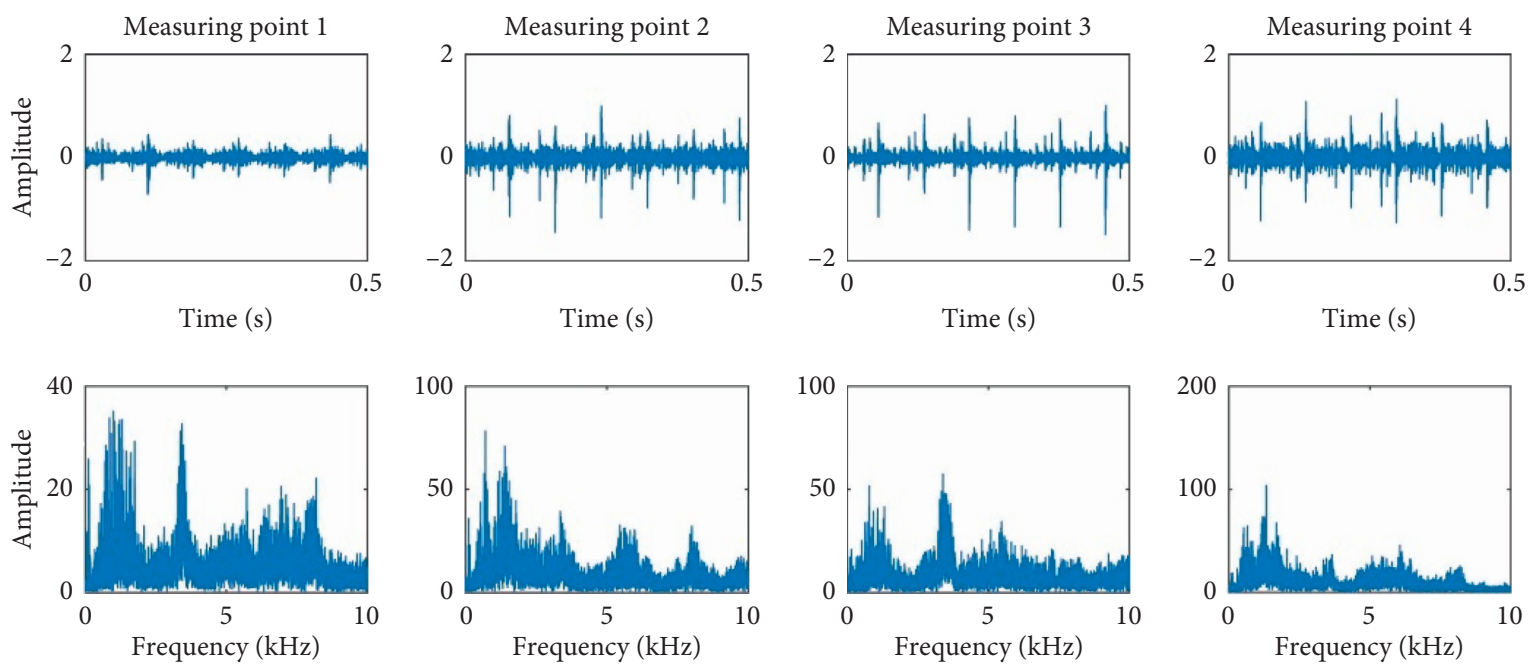

FIgURE 10: The waveforms of original signals under left 1-cylinder (time domain and frequency domain).

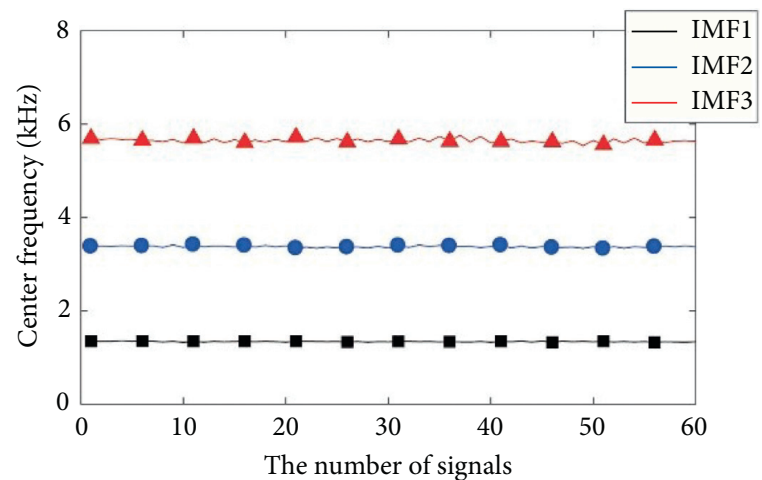

(a)

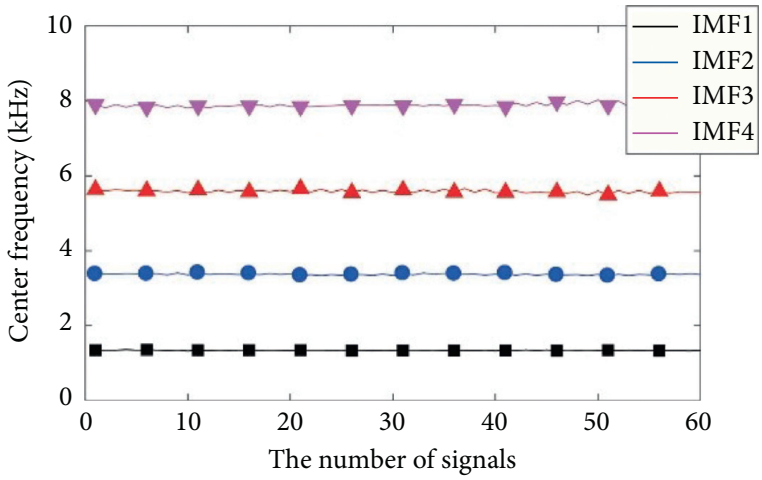

(b)

Figure 11: Continued. 


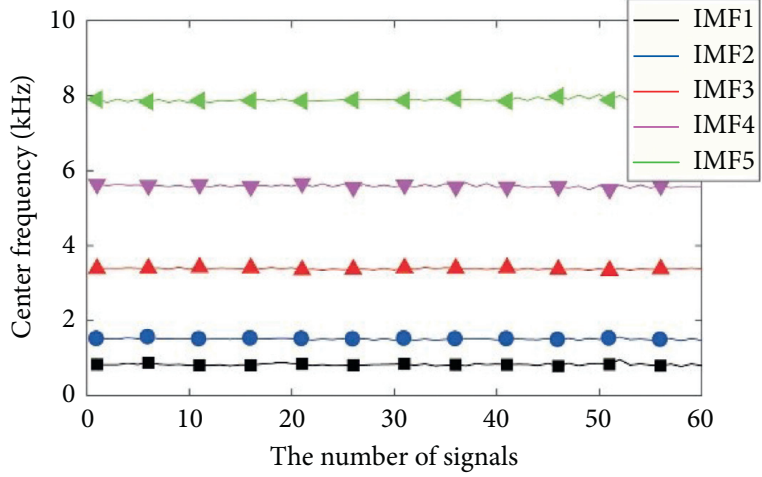

(c)

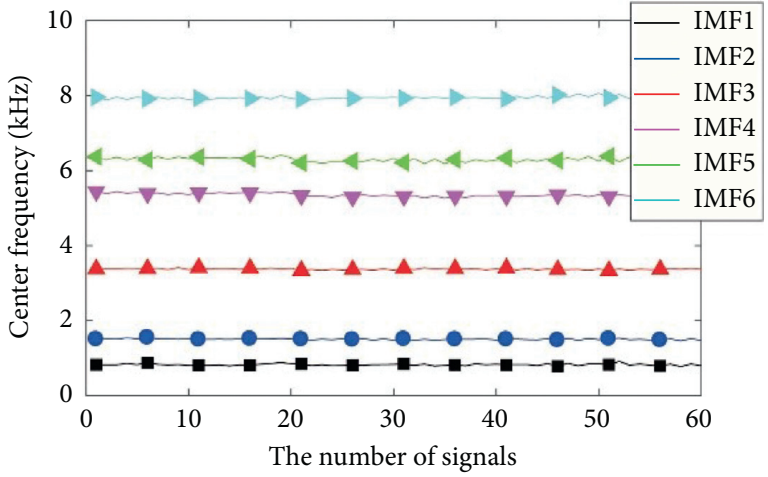

(d)

Figure 11: Center frequency distribution of 60 samples under normal state. (a) $K=3$. (b) $K=4$. (c) $K=5$. (d) $K=6$.

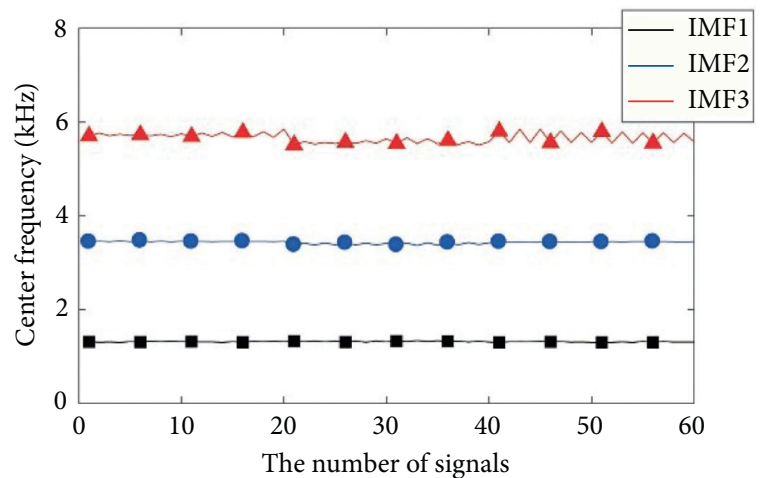

(a)

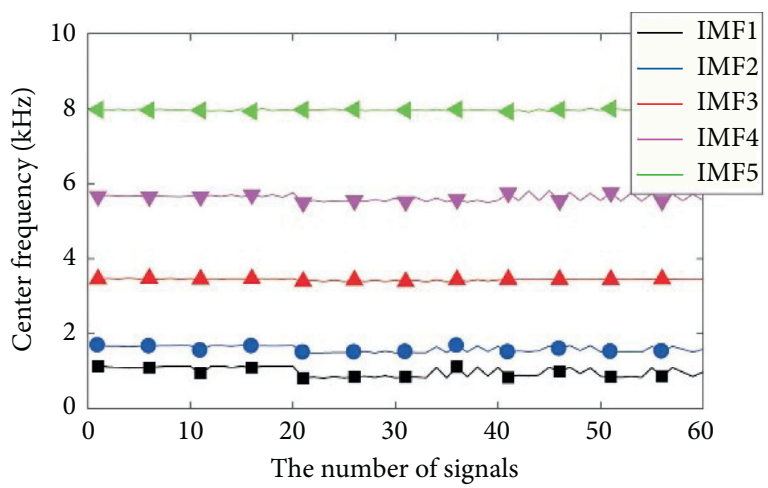

(c)

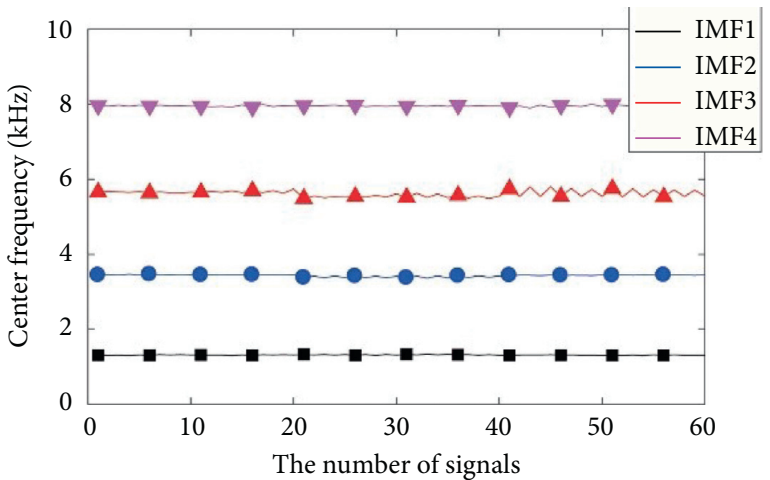

(b)

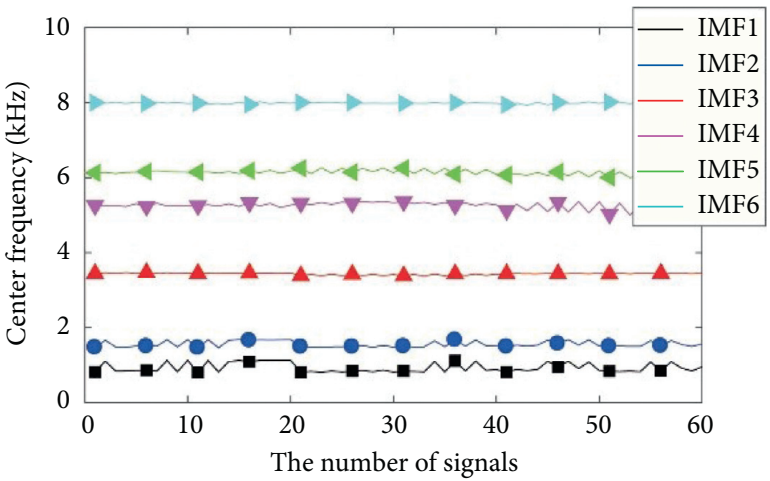

(d)

Figure 12: enter frequency distribution of 60 samples under left 1 -cylinder misfire. (a) $K=3$. (b) $K=4$. (c) $K=5$. (d) $K=6$.

kernel function is the main factor determining the performance of SVM; RBF kernel function is selected for fault diagnosis due to its excellent performance on nonlinear classification problems. The training results and test results are shown in Tables 4 and 5. As can be seen, both the training sample and the test sample have a high accuracy rate, of which the training sample accuracy rate is $99.3 \%$, and the test sample accuracy rate reaches $100 \%$. As a result, the band energy value of each component obtained by MVMD decomposition can be used as characteristic parameter for fault identification.

The vibration signals of the cylinder head are greatly affected by the working conditions of the diesel engine, which in turn affects the accuracy of fault diagnosis. In order to obtain the best diagnostic operating conditions, the above six operating conditions are used to extract band energy according to the method proposed in this paper and are input into the SVM for fault identification. Table 6 shows 

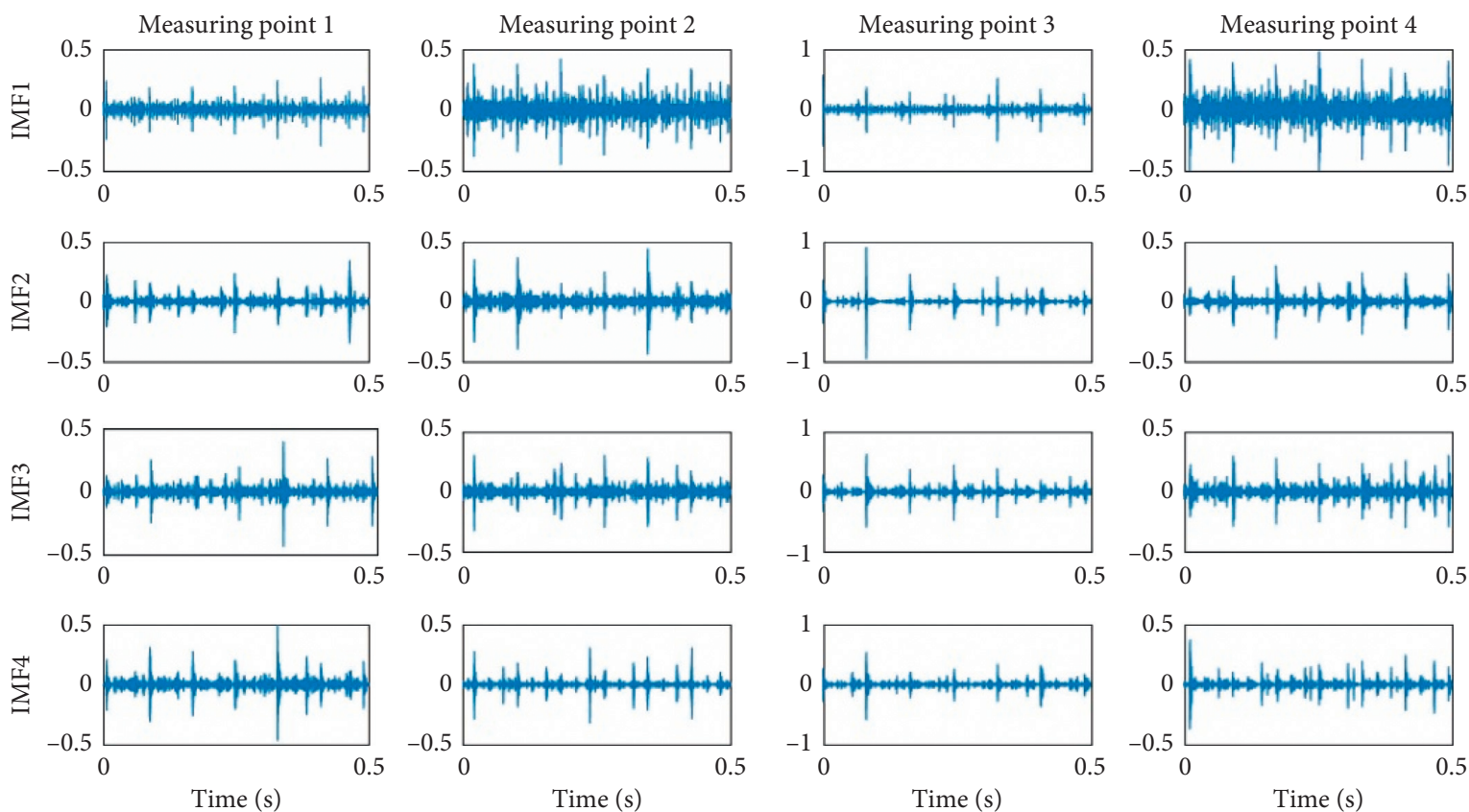

FIGURE 13: Decomposition result of original signals by MVMD under normal state.
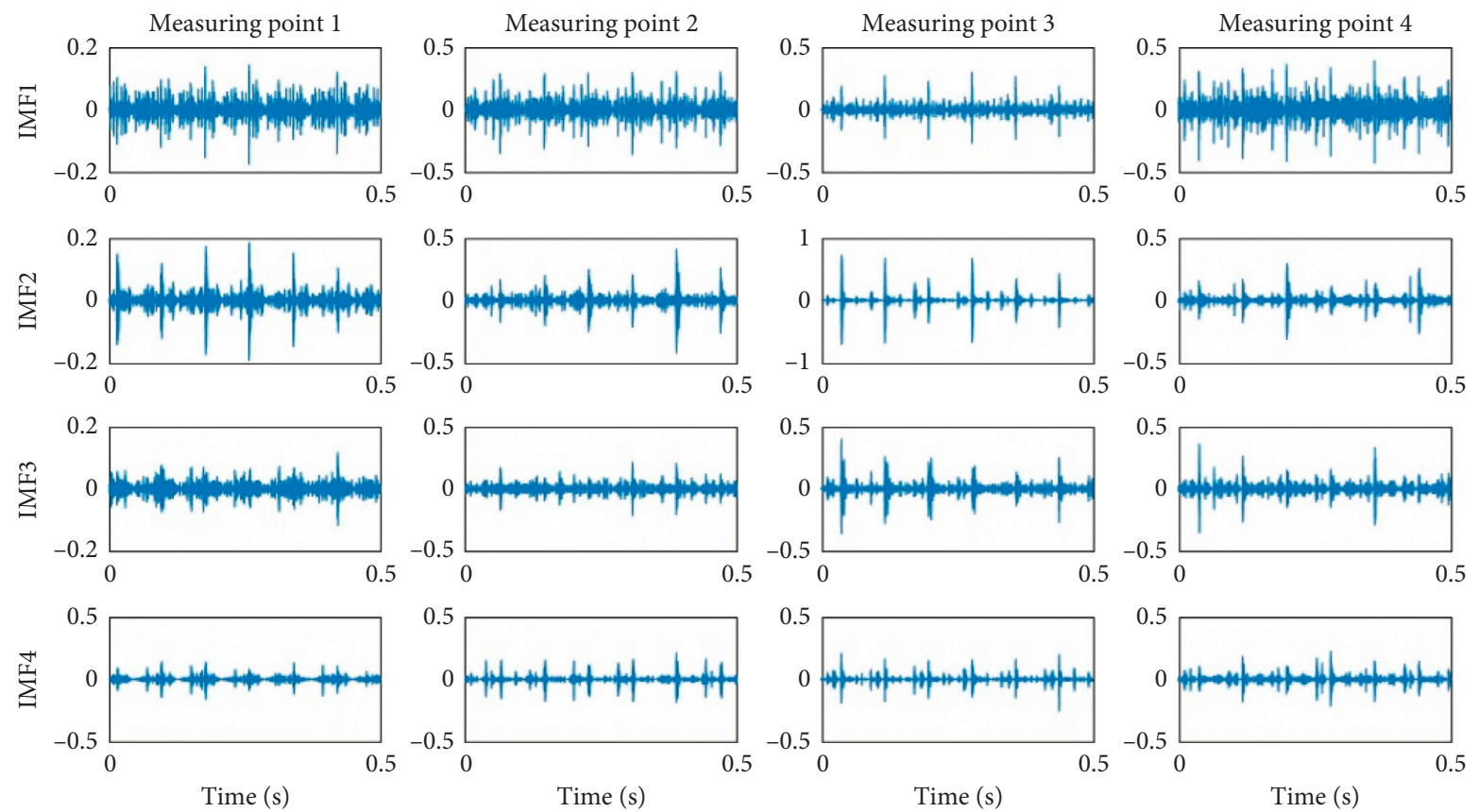

FIgURE 14: Decomposition result of original signals by MVMD under left 1-cylinder misfire.

TABLE 3: Energy values of 4 IMF components at each measuring point under different states.

\begin{tabular}{|c|c|c|c|c|c|c|c|c|c|c|c|c|c|c|c|c|c|}
\hline \multirow{2}{*}{ No } & \multicolumn{4}{|c|}{ IMF of position 1} & \multicolumn{4}{|c|}{ IMF of position 2} & \multicolumn{4}{|c|}{ IMF of position 3} & \multicolumn{4}{|c|}{ IMF of position 4} & \multirow{2}{*}{ Label } \\
\hline & 1 & 2 & 3 & 4 & 1 & 2 & 3 & 4 & 1 & 2 & 3 & 4 & 1 & 2 & 3 & 4 & \\
\hline 1 & 13.5 & 46.0 & 23.5 & 62.0 & 13.8 & 17.0 & 24.6 & 11.4 & 12.6 & 15.5 & 28.0 & 16.6 & 15.1 & 9.0 & 22.0 & 11.0 & 1 \\
\hline 2 & 13.0 & 52.8 & 21.0 & 66.8 & 11.1 & 15.5 & 31.9 & 17.4 & 10.1 & 16.0 & 25.1 & 17.2 & 14.6 & 9.8 & 16.1 & 11.0 & 1 \\
\hline 3 & 12.5 & 46.8 & 25.4 & 63.1 & 13.7 & 18.1 & 27.3 & 11.4 & 12.4 & 15.9 & 27.7 & 17.0 & 15.9 & 9.1 & 21.6 & 10.9 & 1 \\
\hline $\begin{array}{l}\cdots \\
61\end{array}$ & 6.8 & 34.9 & 10.5 & 51.1 & 5.2 & 15.1 & 41.0 & 9.0 & 2.7 & 7.6 & 11.8 & 10.7 & 3.9 & 5.5 & 4.7 & 6.4 & 2 \\
\hline 62 & 6.4 & 36.3 & 11.6 & 50.3 & 8.0 & 12.4 & 27.7 & 11.4 & 2.7 & 10.6 & 8.5 & 15.2 & 4.1 & 7.2 & 3.6 & 5.5 & 2 \\
\hline 63 & 6.8 & 35.6 & 11.3 & 51.6 & 5.3 & 15.6 & 40.7 & 9.2 & 2.7 & 7.9 & 12.2 & 11.1 & 4.1 & 6.1 & 4.6 & 6.3 & 2 \\
\hline
\end{tabular}


TABLE 4: Training sample SVM classification result.

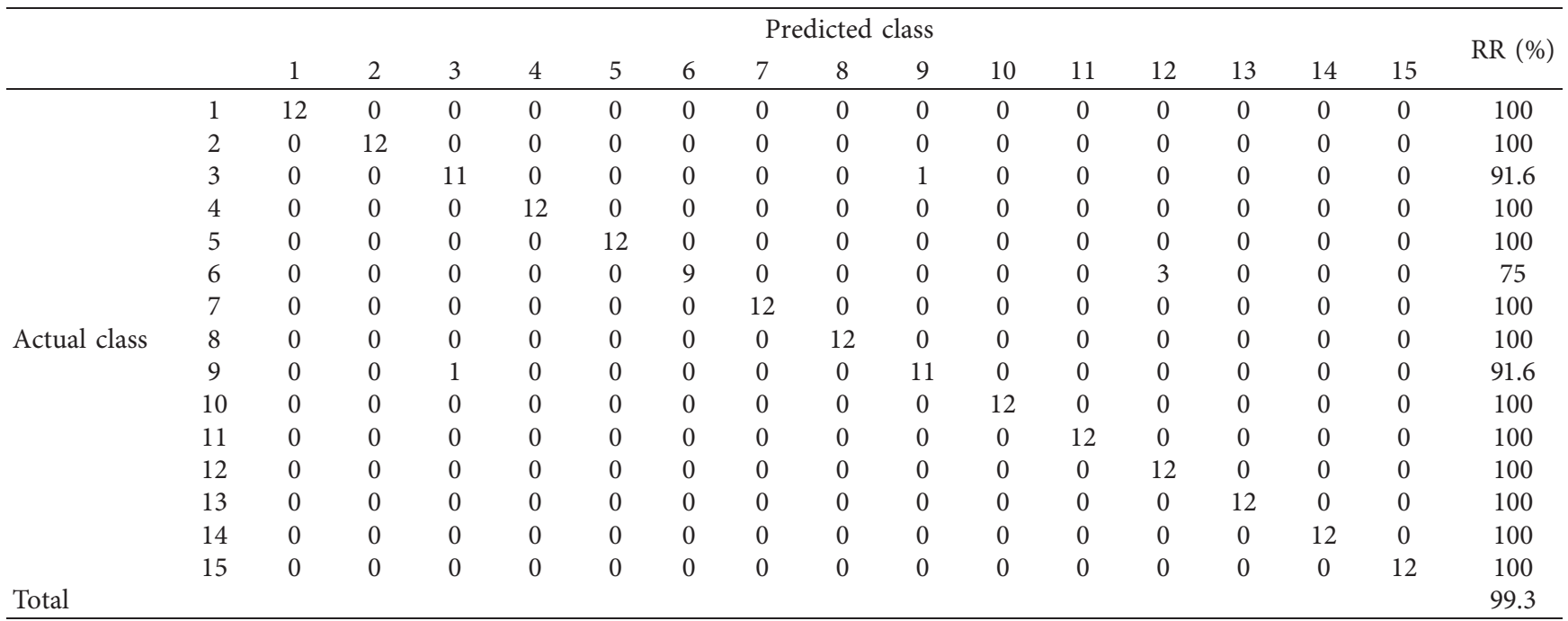

TABle 5: Test sample SVM classification result.

\begin{tabular}{|c|c|c|c|c|c|c|c|c|c|c|c|c|c|c|c|c|c|}
\hline & \multicolumn{16}{|c|}{ Predicted class } & \multirow{2}{*}{$\mathrm{RR}(\%)$} \\
\hline & & 1 & 2 & 3 & 4 & 5 & 6 & 7 & 8 & 9 & 10 & 11 & 12 & 13 & 14 & 15 & \\
\hline \multirow{15}{*}{ Actual class } & 1 & 12 & 0 & 0 & 0 & 0 & 0 & 0 & 0 & 0 & 0 & 0 & 0 & 0 & 0 & 0 & 100 \\
\hline & 2 & 0 & 12 & 0 & 0 & 0 & 0 & 0 & 0 & 0 & 0 & 0 & 0 & 0 & 0 & 0 & 100 \\
\hline & 3 & 0 & 0 & 12 & 0 & 0 & 0 & 0 & 0 & 0 & 0 & 0 & 0 & 0 & 0 & 0 & 100 \\
\hline & 4 & 0 & 0 & 0 & 12 & 0 & 0 & 0 & 0 & 0 & 0 & 0 & 0 & 0 & 0 & 0 & 100 \\
\hline & 5 & 0 & 0 & 0 & 0 & 12 & 0 & 0 & 0 & 0 & 0 & 0 & 0 & 0 & 0 & 0 & 100 \\
\hline & 6 & 0 & 0 & 0 & 0 & 0 & 12 & 0 & 0 & 0 & 0 & 0 & 0 & 0 & 0 & 0 & 100 \\
\hline & 7 & 0 & 0 & 0 & 0 & 0 & 0 & 12 & 0 & 0 & 0 & 0 & 0 & 0 & 0 & 0 & 100 \\
\hline & 8 & 0 & 0 & 0 & 0 & 0 & 0 & 0 & 12 & 0 & 0 & 0 & 0 & 0 & 0 & 0 & 100 \\
\hline & 9 & 0 & 0 & 0 & 0 & 0 & 0 & 0 & 0 & 12 & 0 & 0 & 0 & 0 & 0 & 0 & 100 \\
\hline & 10 & 0 & 0 & 0 & 0 & 0 & 0 & 0 & 0 & 0 & 12 & 0 & 0 & 0 & 0 & 0 & 100 \\
\hline & 11 & 0 & 0 & 0 & 0 & 0 & 0 & 0 & 0 & 0 & 0 & 12 & 0 & 0 & 0 & 0 & 100 \\
\hline & 12 & 0 & 0 & 0 & 0 & 0 & 0 & 0 & 0 & 0 & 0 & 0 & 12 & 0 & 0 & 0 & 100 \\
\hline & 13 & 0 & 0 & 0 & 0 & 0 & 0 & 0 & 0 & 0 & 0 & 0 & 0 & 12 & 0 & 0 & 100 \\
\hline & 14 & 0 & 0 & 0 & 0 & 0 & 0 & 0 & 0 & 0 & 0 & 0 & 0 & 0 & 12 & 0 & 100 \\
\hline & 15 & 0 & 0 & 0 & 0 & 0 & 0 & 0 & 0 & 0 & 0 & 0 & 0 & 0 & 0 & 12 & 100 \\
\hline Total & & & & & & & & & & & & & & & & & 100 \\
\hline
\end{tabular}

that the method proposed in this paper shows a high accuracy rate for fire faults under six operating conditions, of which the accuracy rate is the highest under the $1500 \mathrm{rpm}$ and 50\% load operating condition. The vibration signals generated by a diesel engine at low speed and low load are small, which causes its band energy characteristics to be easily overwhelmed by noise. With the increase of the speed and load, the vibration signal with fault characteristics is more likely to expose the misfire fault, but it will also cause an increase in noise. Therefore, the recognition rate of misfire faults is low at low speed and low load. As the speed increases, the recognition rate increases. The recognition rate is highest at medium speed, but it decreases a little at high speed.

In order to verify the superiority of the method proposed in this paper, four other common methods are used to extract energy from the same set of data (operating conditions at $1500 \mathrm{rpm}$ and 50\% load), and the energy values are put into the SVM for misfire diagnosis and identification. The four methods are the original signal energy method, the VMD band energy method, the MEMD band energy method, and the NAMEMD band energy method. Among them, the original signal energy method uses the energy values of the four original measurement points as the fault characteristic parameters. The VMD frequency band energy method is to decompose a single-channel signal (vibration signal from measuring point 1) and calculate the energy value of each frequency band as a fault characteristic parameter; similarly, the number of modes $K$ is 4 . The MEMD band energy method is to decompose the original signal and calculate the band energy values of the component with the larger correlation coefficient of the original signal as the fault characteristic parameter (the first 4th-order components have greater correlation with the original signal after 
TABLE 6: The recognition rate effects under different operating conditions.

\begin{tabular}{lcccccc}
\hline & \multicolumn{2}{c}{$800 \mathrm{rpm}$} & \multicolumn{2}{c}{$1500 \mathrm{rpm}$} & \multicolumn{2}{c}{$2200 \mathrm{rpm}$} \\
& No load (\%) & $50 \%$ load (\%) & No load (\%) & $50 \%$ load (\%) & No load (\%) & $50 \%$ load (\%) \\
\hline Training sample & 83.33 & 98.88 & 99.72 & 99.31 & 98.47 & 98.75 \\
Test sample & 80.55 & 98.33 & 99.44 & 100 & 99.44 & 97.22 \\
\hline
\end{tabular}

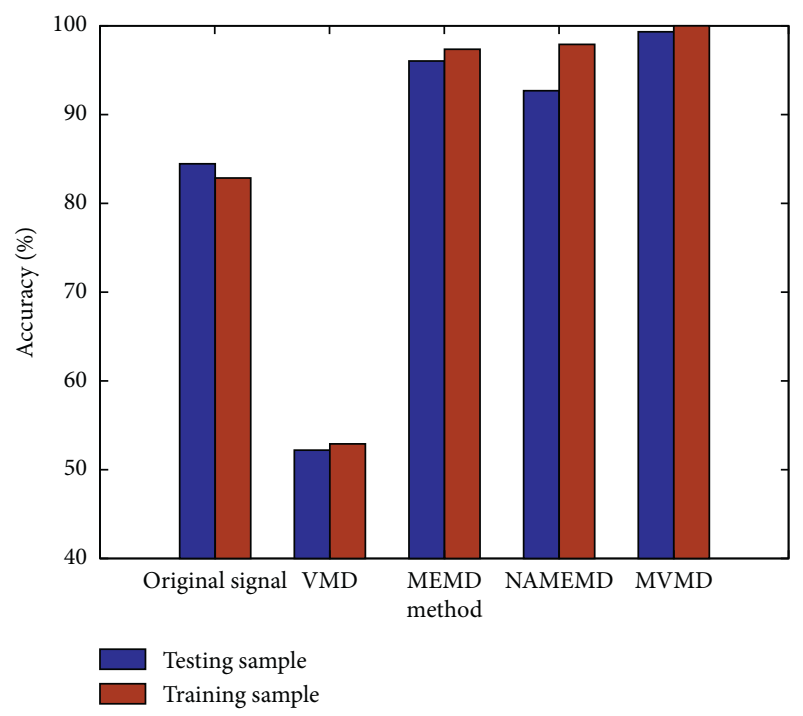

FIGURE 15: Comparison of different methods classification results.

calculation). The NAMEND band energy method has the same steps as the MEMD band energy method, and the number of noise signals added during the decomposition process is 2 .

The recognition rates of different methods for the training set and test set are shown in Figure 15. It can be seen in the figure that the method for extracting band energy using multivariate adaptive decomposition is more accurate than the other two methods in identifying misfires. Among all the methods, the MVMD band energy method has the highest accuracy rate for identifying faults, reaching almost $100 \%$.

\section{Conclusions and Discussion}

In this paper, Multivariate Variational Mode Decomposition is introduced into the field of mechanical fault diagnosis, and a novel fault diagnosis method for diesel engine based on MVMD and band energy is presented. The simulation test and bench test were used to study the MVMD adaptive decomposition diesel engine multivariate vibration signals, and the following conclusions were obtained:

(1) Characteristic frequency in multicomponent modulation signals can be extracted by MVMD effectively. Compared with MEMD and NAMEMD, MVMD algorithm performs better in suppressing modal aliasing and endpoint effects and has higher decomposition efficiency.

(2) Vibration signals at four positions of the diesel engine cylinder head were collected through bench tests, and misfire fault identification was performed by the MVMD band energy method. The results show that the training and test sets have high fault recognition rates. Different operating conditions have a certain degree of impact on the fault recognition rate, and the highest fault recognition rate is at $1500 \mathrm{rpm}$ and 50\% load. Compared with the original signal energy method, the VMD band energy method, the MEMD band energy method, and the NAMEMD band energy method, the fault diagnosis method based on the MVMD band energy method performs best.

(3) Impacts of misfire faults are differentiated by various frequency bands at each measuring point. Multivariate signals contain more comprehensive information about mechanical equipment, which is more suitable for state assessment and fault diagnosis. The application of multivariate signals adaptive decomposition is more worthy of study.

However, there are some problems to be solved in following work:

(1) There are several shortcomings in the setting of fault types:

The misfire failure type of multicylinder is not sufficiently considered. For example, according to the distance between two misfired cylinders, it can be divided into far cylinder misfire with distance of 1 (L1 cylinder \& L4 cylinder), far cylinder misfire with distance of 2 (L1 cylinder \& R5 cylinder), and so on.

There are fewer types of working conditions. As the load increases, the higher the power performance of the diesel engine is required, and the misfire failure has a greater impact on the power performance. Therefore, this paper only performs the two cases of $50 \%$ load and no load, which are limited to the test. Full-load conditions and other conditions tests should also be considered.

This paper only discusses misfire faults. As complex machine with many parts, the diesel engine has many typical faults, such as too large/small clearance between the inlet and exhaust valves, oil leakage from the injector, and so on, which has a greater impact on performance index of the diesel engine.Next, we need to conduct more experiments to enrich the types of failures.

(2) During the test, only one diesel engine on the test bench was carried out. All data are obtained from this diesel engine, and no consideration is given to 
whether it can be generalized to different diesel engines of the same model, different models of diesel engines, and diesel engines on real vehicles. In further research, we will consider enriching the test objects to verify the generalization ability of the algorithm, which is of great significance for its application to engineering practice.

(3) The MVMD algorithm focuses on the global concept and lacks local thoughts, which is also a common defect of all adaptive decomposition algorithms. During the operation of the diesel engine, some faults only have a large impact on the vibration signal of a certain period of time, while other periods are almost unaffected. Sometimes, the partial information of the fault is covered up if the signal of the entire time period is processed. How to focus adaptively on the fault impact part and highlight the fault characteristics is also a problem that should be focused on in the next step.

\section{Data Availability}

The data used to support the findings of this study are available from the corresponding author upon request.

\section{Conflicts of Interest}

The authors declare that they have no conflicts of interest.

\section{Authors' Contributions}

Cheng $\mathrm{Gu}$ proposed methods and wrote the manuscript; Xinyong Qiao designed the experiment and provided academic guidance; Ying Jin and Yanbin Liu conducted experiments and analyzed the data. All authors discussed the results and commented on the manuscript.

\section{Acknowledgments}

This work was funded by the National Basic Research Program of China (grant no. 6312520302).

\section{References}

[1] J. M. Liu, Research on the Method for Evaluation and Predicting the Technical States of Armored Vehicle Diesel engine, Academy of Armored Force Engineering, Beijing, China, 2006.

[2] Y. P. Shi, Analysis of Diesel Engine Cylinder Head Vibration Signal and Application in Fault diagnosis, Academy of Armored Force Engineering, Beijing, China, 2011.

[3] L. Arnone, M. Boni, S. Manelli, O. Chiavola, S. Conforto, and E. Recco, "Diesel engine combustion monitoring through block vibration signal analysis," in SAE Technical Paper, SAE International, Warrendale, PA, USA, 2009.

[4] O. Chiavola, G. Chiatti, L. Arnone, and S. Manelli, "Combustion characterization in diesel engine via block vibration analysis," in SAE Technical Paper, p. 9, SAE International, Warrendale, PA, USA, 2010.

[5] S. Liu, F. Gu, and A. Ball, "Detection of engine valve faults by vibration signals measured on the cylinder head," Proceedings of the Institution of Mechanical Engineers, Part D: Journal of Automobile Engineering, vol. 220, no. 3, pp. 379-386, 2006.

[6] N. E. Huang, Z. Shen, S. R. Long et al., "The empirical mode decomposition and the Hilbert spectrum for nonlinear and non-stationary time series analysis," Proceedings of the Royal Society of London. Series A: Mathematical, Physical and Engineering Sciences, vol. 454, no. 1971, pp. 903-995, 1998.

[7] Y. Li, P. W. Tse, X. Yang, and J. Yang, "EMD-based fault diagnosis for abnormal clearance between contacting components in a diesel engine," Mechanical Systems and Signal Processing, vol. 24, no. 1, pp. 193-210, 2010.

[8] Z. Wu and N. E. Huang, "Ensemble empirical mode decomposition: a noise-assisted data analysis method," Advances in Adaptive Data Analysis, vol. 1, no. 1, pp. 1-41, 2009.

[9] X. Wang, C. Liu, F. Bi, X. Bi, and K. Shao, "Fault diagnosis of diesel engine based on adaptive wavelet packets and EEMDfractal dimension," Mechanical Systems and Signal Processing, vol. 41, no. 1-2, pp. 581-597, 2013.

[10] J. S. Smith, “The local mean decomposition and its application to EEG perception data," Journal of the Royal Society Interface, vol. 2, no. 5, pp. 443-454, 2005.

[11] K. Dragomiretskiy and D. Zosso, "Variational mode decomposition," IEEE Transactions on Signal Processing, vol. 62, no. 3, pp. 531-544, 2013.

[12] J. Yao, Y. Xiang, S. Qian, S. Wang, and S. Wu, "Noise source identification of diesel engine based on variational mode decomposition and robust independent component analysis," Applied Acoustics, vol. 116, pp. 184-194, 2017.

[13] X. Bi, S. Cao, and D. Zhang, "Diesel engine valve clearance fault diagnosis based on improved variational mode decomposition and bispectrum," Energies, vol. 12, no. 4, p. 661, 2019.

[14] E. Song, Y. Ke, C. Yao, Q. Dong, and L. Yang, "Fault diagnosis method for high-pressure common rail injector based on IFOA-VMD and hierarchical dispersion entropy," Entropy, vol. 21, no. 10, p. 923, 2019.

[15] X. Bi, S. Cao, and D. Zhang, "A variety of engine faults detection based on optimized variational mode decompositionrobust independent component analysis and Fuzzy C-mean clustering," IEEE Access, vol. 7, pp. 27756-27768, 2019.

[16] Z. Li, X. Yan, Z. Guo, Y. Zhang, and Z. Peng, "Condition monitoring and fault diagnosis for marine diesel engines using information fusion techniques," Elektronika Ir Elektrotechnika, vol. 123, no. 7, pp. 109-112, 2012.

[17] J. Tao, Y. Liu, and D. Yang, "Bearing fault diagnosis based on deep belief network and multi-sensor information fusion," Shock and Vibration, vol. 2016, Article ID 9306205, 9 pages, 2016.

[18] J. Hang, M. Cheng, and J. Zhang, "Fault diagnosis of wind turbine based on multi-sensors information fusion technology," IET Renewable Power Generation, vol. 8, no. 3, pp. 289-298, 2014.

[19] T. Tanaka and D. P. Mandic, "Complex empirical mode decomposition,” IEEE Signal Processing Letters, vol. 14, no. 2, pp. 101-104, 2007.

[20] G. Rilling, P. Flandrin, P. Gonçalves, and J. M. Lilly, "Bivariate empirical mode decomposition," IEEE Signal Processing Letters, vol. 14, no. 12, pp. 936-939, 2007.

[21] N. Ur. Rehman and D. P. Mandic, "Empirical mode decomposition for trivariate signals," IEEE Transactions on Signal Processing, vol. 58, no. 3, pp. 1059-1068, 2010.

[22] N. Rehman and D. P. Mandic, "Multivariate empirical mode decomposition," Proceedings of the Royal Society A: 
Mathematical, Physical and Engineering Sciences, vol. 466, no. 2117, pp. 1291-1302, 2010.

[23] Y. Lv, R. Yuan, and G. Song, "Multivariate empirical mode decomposition and its application to fault diagnosis of rolling bearing," Mechanical Systems and Signal Processing, vol. 81, pp. 219-234, 2016.

[24] N. U. Rehman and D. P. Mandic, "Filter bank property of multivariate empirical mode decomposition," IEEE Transactions on Signal Processing, vol. 59, no. 5, pp. 2421-2426, 2011.

[25] Z. Wu, S.-P. Yang, B. Ren, X.-N. Ma, and J.-C. Zhang, "Rolling element bearing fault diagnosis method based on NAMEMD and multi-scale morphology," Journal of Vibration and Shock, vol. 35, no. 4, pp. 127-133, 2016.

[26] N. U. Rehman and H. Aftab, "Multivariate variational mode decomposition," IEEE Transactions on Signal Processing, vol. 67, no. 23, pp. 6039-6052, 2019. 